\title{
Long-range migration of centrioles to the apical surface of the olfactory epithelium
}

Kaitlin Ching ${ }^{1,2,+}$, Jennifer T. Wang ${ }^{1,+}$, Tim Stearns ${ }^{1,3, *}$

${ }^{1}$ Department of Biology, Stanford University, Stanford, CA 94305, USA

${ }^{2}$ Department of Molecular, Cell, and Developmental Biology, University of California, Los Angeles, CA 90095, USA

${ }^{3}$ Department of Genetics, Stanford University School of Medicine, Stanford, CA 94305, USA

*Correspondence: stearns@stanford.edu

${ }^{+}$Authors contributed equally to this work

\section{Abstract}

Olfactory sensory neurons (OSNs) in vertebrates detect odorants using multiple cilia, which protrude from the end of the dendrite and require centrioles for their formation. In mouse olfactory epithelium, the centrioles originate in progenitor cells near the basal lamina, often 50 to $100 \mu \mathrm{m}$ from the apical surface. It is unknown how centrioles traverse this distance or mature to form cilia. Using high-resolution expansion microscopy, we found that centrioles migrate together, with multiple centrioles per group and multiple groups per OSN, during dendrite outgrowth. Centrioles were found by live imaging to migrate slowly, with a maximum rate of 0.18 $\mu \mathrm{m} / \mathrm{min}$. Centrioles in migrating groups were associated with microtubule nucleation factors, but acquired rootletin and appendages only in mature OSNs. The parental centriole had preexisting appendages, formed a single cilium prior to other centrioles, and retained its unique appendage configuration in the mature OSN. We developed an air-liquid interface explant culture system for OSNs and used it to show that centriole migration can be perturbed ex vivo by stabilizing microtubules. We consider these results in the context of a comprehensive model for centriole formation, migration, and maturation in this important sensory cell type.

keywords: centriole, centrosome, cilium, dendrite, expansion microscopy, migration, olfaction, OSN, sensory neuron

\section{Introduction}

Chemosensation in many animals is mediated by ciliated neurons that have a sensory cilium at the end of a dendrite, positioned to sense the external environment. In vertebrates, olfactory sensory neurons (OSNs), found within the olfactory epithelium, have multiple cilia in a dendritic knob exposed to odorants (Cuschieri \& Bannister, 1975, Menco 1997, McEwen et al., 2008, Oberland \& Neuhaus, 2014). The structure and function of these cilia are critical to olfaction. 
They provide a specialized membrane area for signaling via G protein coupled olfactory receptors and disruption of cilium structure and function can result in anosmia (Jenkins et al., 2009, Kulaga et al., 2004, McEwen et al., 2007). The microtubule structure of each cilium is templated by a centriole at its base, which is also referred to as a basal body. Centrioles in many cell types also recruit pericentriolar material (PCM) to form the centrosome, the major

43 microtubule organizing center.

To create the requisite number of olfactory cilia, cells must amplify centrioles from an initial pair. We previously demonstrated that centriole amplification occurs in mitotically active progenitor cells located near the basal lamina of the olfactory epithelium in mice (Ching \& Stearns, 2020). This is unique among cells with multiple cilia, as epithelial cells with multiple motile cilia in the trachea, oviduct, and brain ependyma (multiple motile cilia cells, MMCCs) amplify centrioles only after mitosis is complete.

Progenitor cells generate OSNs during embryonic development and throughout adult life, continuing to generate new ciliated olfactory neurons long after the epithelium has been established (Moulton \& Beidler, 1967; Mulvaney \& Heist, 1971). As a daughter cell from the progenitor division differentiates into an immature OSN, it extends projections in opposite directions: a dendrite toward the apical surface to detect odorants, and an axon through the basal lamina toward the olfactory bulb. In vivo fate mapping in mouse has shown that this process begins one day after the progenitor's last cell cycle (Rodriguez-Gil et al., 2015). During neurite extension, the cell body of the neuron remains close to the basal lamina (Fig 1A) (Rodriguez-Gil et al., 2015).

Centrioles are amplified in the cell body and must migrate to the apical surface, traversing a distance of 50 to $100 \mu \mathrm{m}$ or more (Fig 1A). Using transmission electron microscopy, migrating centrioles have been previously observed in mammalian olfactory sensory neurons (Heist \& Mulvaney, 1968; Mulvaney \& Heist, 1971). Within the thin, 1- to 2- $\mu \mathrm{m}$-wide dendrites, centrioles were found to be grouped, with more groups being found in the most apical portion of the

67 epithelium (Mulvaney \& Heist, 1971). Six days after the progenitor cell's last cell cycle, immature

68 OSNs differentiate into mature OSNs and express markers of maturation, such as olfactory 69 marker protein (OMP) (Rodriquez-Gil et al., 2015). Upon maturation, they form multiple cilia at 70 the apical end of the dendrite, and their nuclei migrate towards the apical surface in the days to 71 weeks that follow (Rodriguez-Gil et al., 2015). 
73 For OSNs, centrioles must be moved to the apical surface of the olfactory epithelium to

74 assemble sensory cilia where they will have access to odorants. Many cell types across a

75 variety of organisms are known to relocate or reorient their centrioles, including ciliating

76 vertebrate cells (Dawe et al., 2007), Drosophila nurse cells in the germarium (Mahowald \&

77 Strassheim, 1970; Bolivar et al., 2001), and macrociliary cells in the comb jelly Beroe (Tamm \&

78 Tamm, 1988). Perhaps the most relevant example is the C. elegans PQR neuron, in which a

79 single centriole migrates approximately $5 \mu \mathrm{m}$ along the dendrite by the activity of the minus-end 80 directed microtubule motor, dynein (Li et al., 2017). In contrast to the PQR neuron, vertebrate

81 OSNs each contain multiple centrioles, move those centrioles over a 10-fold greater distance,

82 and develop throughout the life of the animal, which frequently requires centrioles to migrate

83 through an established epithelium. Despite these biologically interesting challenges,

84 mechanisms of movement and the molecular composition of migrating OSN centrioles remain unclear.

Here, we develop new techniques for imaging and manipulating OSNs and use these to explore the mechanisms by which multiple centrioles migrate and become competent to form the multiple cilia that are important for the sensory function of OSNs. Our findings inform our understanding of OSN cell biology, as well as long-range organelle movement more broadly.

\section{Results}

Identification of migrating and mature centrioles in the olfactory epithelium.

96 The olfactory epithelium is composed of multiple cell types, including olfactory sensory neurons 97 (OSNs) and non-neuronal cell types known as sustentacular cells (Fig 1A). To investigate the 98 migration of OSN centrioles, we observed their localization in fixed olfactory epithelium taken 99 from mice expressing eGFP-centrin2, a component of centrioles (Bangs et al., 2015). OSNs are 100 generated from progenitors throughout development and adult life, and we chose to use adult 101 mice in these experiments to enable observation of centriole migration across the full depth of 102 the mature olfactory epithelium. En face imaging of the apical surface allowed us to identify 103 dendritic knobs ensheathed in sustentacular cells with borders rich in filamentous actin (Fig 1B, 104 1C) (Liang, 2018). In side views of the olfactory epithelium, migrating centrioles were identified 
as elongated clusters of puncta, in accordance with previous reports (Fig 1D) (Heist \& Mulvaney, 1968; Klimenkov et al., 2018; Mulvaney \& Heist, 1971, Ying et al., 2014).

To better visualize centriole migration in OSNs at higher resolution we applied expansion microscopy (Gambarotto et al., 2019, Sahabandu et al., 2019) to sections of adult olfactory epithelium. We were able to reproducibly expand these samples approximately 4-fold, and could resolve olfactory cilia in mature OSNs, as well as migrating centrioles in immature OSNs (Fig 1E, 1F) by staining for acetylated tubulin, which marks centrioles, cilia, and, more faintly, the microtubules of the dendrite. En face imaging of the dendritic knob using expansion microscopy identified groups of centrioles at the apical surface (Fig 1E). Side views of the olfactory epithelium identified groups of migrating centrioles positioned below the apical surface (Fig 1F).

With the increased resolution afforded by expansion microscopy, we counted centriole number in progenitor cells and mature OSNs by staining for acetylated tubulin and using a semiautomated volume segmentation and counting pipeline (Fig 1 - Supplementary Fig 1A, B). We found that basally-positioned progenitor cells with amplified centrioles harbored, on average, 26.40 centrioles $(\mathrm{SEM}=2.234$; standard deviation $=9.992 ; \mathrm{n}=20$ cells from 2 mice, Fig 1 Supplementary Fig 1C). In mature OSNs that had completed centriole migration with centrioles docked at the dendritic knob, each cell had, on average, 36.46 centrioles (SEM = 1.665; standard deviation = 8.156, $\mathrm{n}=24$ dendritic knobs from 2 mice, Fig 1 - Supplementary Fig 1C). These results suggest that progenitor cells form the majority of centrioles present in the dendritic knob of mature OSNs, in keeping with our previously published work (Ching \& Stearns 2020).

To determine whether all of the centrioles at mature dendritic knobs nucleate cilia, we counted the number of cilia found in each dendritic knob (Figure 1 - Supplementary Fig 1B, 1D). We found that, on average, $85 \%$ of centrioles at a dendritic knob were associated with cilia $(\mathrm{SEM}=2.47$; standard deviation $=12.10)$, with an average of 30.54 cilia per knob $(\mathrm{SEM}=1.455$; standard deviation $=7.126$ ). It is unclear whether the $15 \%$ of centrioles not associated with a cilium previously had a cilium, will eventually make a cilium, or whether they are excess to an independent process that determines cilium number. 

dendrite outgrowth.

140 The dendrites of mature OSNs extend from the cell body in the basal region of the olfactory 141 epithelium to the ciliated dendritic knobs at the apical surface. In our expansion microscopy 142 images, we noticed that centrioles within dendrites were often grouped together (Fig 1F). We

143 first tested whether multiple groups of centrioles could be present within a single dendrite, as 144 suggested by previous TEM results (Heist \& Mulvaney, 1968; Mulvaney \& Heist, 1971). We 145 imaged immature OSNs using expansion microscopy, through the entire thickness of the 146 dendrite. We found that centrioles were positioned in multiple groups within the same dendrite (Fig 2A).

We next asked whether the dendrite completely extends prior to centriole migration, or whether migration occurs in tandem with dendrite extension. We imaged growth-associated protein 43 (GAP43), a marker of immature OSNs and some progenitor cells, to observe the extent of dendrites during outgrowth (Mclntyre et al., 2010). We observed centrioles in partially elongated dendrites with growth cones, indicating that the dendrite does not fully extend prior to centriole migration (Fig 2B). By probing for $\beta$-tubulin III (TUBB3), a tubulin isotype expressed primarily in neurons, we observed that microtubules were present both apical to and basal to the migrating centrioles (Fig 2B). Together, these data indicate that the leading group of centrioles migrates in tandem with dendrite extension and can be followed by lagging groups within the dendrite.

To begin to assess the mechanisms by which centrioles migrate, we established an ex vivo live imaging system for dissected olfactory epithelium. To measure rates of centriole movement in the final stage of migration we used time-lapse confocal microscopy to image migrating eGFPcentrin2-labeled centrioles. Flattened olfactory epithelium was mounted on agarose pads and imaged en face, and z stacks were acquired to track the movement of centriole groups in the apical 15 to $25 \mu \mathrm{m}$ of the epithelium. We found that the cells in the excised olfactory epithelium remained intact with few dying or delaminating cells over the time course of imaging.

166 Considering net movement over the course of the sequences, centriole groups moved slowly, 167 with the fastest moving at approximately $0.18 \mu \mathrm{m}$ per minute $(\mathrm{n}=22$ centriole groups, $\mathrm{N}=3$ 168 animals, Fig 2C, Fig 2 - Supp Vid 1). Most centriole groups exhibited net movement in the 169 apical direction (Fig 2C, Fig 2 - Supp Vid 1, n = 14 out of 22 groups observed), although approximately one third had either net movement in the basal direction or no movement during the imaging period (Fig 2D, Fig 2 - Supp Vid 2, n=8 out of 22 groups observed). 
173 Our results show that centrioles often migrate in groups, moving relatively slowly towards the

174 apical end of the dendrite. How might these groups of centrioles be held together during

175 migration? Centrioles in dividing cells are kept in close proximity by two forms of linkage

176 (Breslow \& Holland 2019; Nigg \& Stearns 2011). Newly formed centrioles are attached to their

177 associated mother centriole by an engagement linker, which is broken by passage through

178 mitosis, and disengaged centrioles are linked by cohesion fibers. Our inability to resolve

179 centriole-centriole relationships in the migrating groups due to tight packing within the narrow

180 dendrite prevents us from being able to determine whether the engagement link is responsible

181 for their grouping; however, we consider it unlikely that all centrioles are held together this way,

182 based on our previous results showing that centriole amplification is initiated prior to mitotic

183 divisions in progenitors (Ching \& Stearns, 2020).

Given that centriole engagement is unlikely to be the major determinant of grouping, we tested whether grouping is mediated by centriole cohesion. Cohesion fibers contain rootletin (CROCC) (Vlijm et al., 2018), which is also a component of the striated rootlet that anchors centrioles bearing cilia (Yang et al., 2002). We performed expansion microscopy to detect rootletin on centrioles. Strikingly, migrating centrioles lacked rootletin signal (Fig 3A), indicating that these groups are not held together by rootletin-based cohesion fibers. We also observed that migrating centrioles not only move in multiple groups, but that some centrioles are not grouped at all, localizing singly between migrating groups in immature OSNs (Fig 3A). The rootletin antibody did detect rootlets associated with centrioles in mature OSNs (Fig 3A) (Menco et al., 1978; McClintock et al., 2008). Thus, rootletin is not present on migrating centrioles and therefore centriole cohesion is unlikely to play a role in grouping centrioles together during migration.

\section{Centriole composition shifts toward maturation during OSN development.}

200 In the typical animal cell cycle, centrioles require two cell cycles after their formation to become 201 competent to form cilia (Breslow \& Holland 2019; Nigg \& Stearns 2011). During this maturation 202 process they lose proteins associated with the early steps of centriole formation, including 203 components of the cartwheel, and acquire proteins associated with microtubule nucleation, as 204 well as distal and subdistal appendages. To understand the progression of centriole maturation 
205 in the olfactory epithelium, we examined proteins associated with centriole formation and

206 microtubule nucleation in progenitors, immature OSNs and mature OSNs.

First, we examined the procentriole markers STIL (SCL/Tal1 interrupting locus protein) and SASS6 (spindle assembly abnormal protein 6) using expansion microscopy. In cycling mammalian cells grown in culture, these proteins are present during procentriole formation in Sphase and are lost from centrioles in mitosis following centriole disengagement (Arquint and

212 Nigg 2014; Strnad et al., 2007). In the olfactory epithelium, both STIL and SASS6 were present

213 on amplifying centrioles in progenitors and localized to the proximal end of newly formed

214 centrioles, supporting the model that these proteins are involved in centriole amplification from

215 rosettes (Fig 3B, 3C). Remarkably, STIL and SASS6 are retained on at least some of the

216 migrating centrioles in immature OSNs and are associated with the presumptive proximal end of 217 these centrioles (Fig 3B, 3C). In mature OSNs, STIL and SASS6 are lost from centrioles (Fig

218 3B, 3C). Together, these results suggest that immature procentrioles are capable of migrating, while the dendritic knob is primarily formed of mature centrioles that lack STIL and SASS6.

In many differentiated neurons, centrioles lose the microtubule nucleating capability that allows them to function as centrosomes. Pericentriolar material (PCM) proteins involved in microtubule organization, including gamma-tubulin, CDK5RAP2 (CDK5 Regulatory Subunit Associated Protein 2) and pericentrin (PCNT), are reduced at centrosomes in these neurons and relocalized to non-centrosomal sites (Leask et al., 1997; Steiss et al., 2010; Yonezawa et al., 2015; Sanchez-Huertas et al., 2016; Wilkes \& Moore, 2020). We were interested in assessing the association of PCM proteins with centrioles in OSNs. We first probed for the microtubule nucleator gamma-tubulin using expansion microscopy. In progenitor cells with amplifying centrioles, gamma-tubulin is present surrounding centrioles (Fig 3D). In immature OSNs, gamma-tubulin localizes to a diffuse cloud around centriole barrels. In mature, fully ciliated OSNs, gamma-tubulin was also present around centrioles. Next, we determined the localization of CDK5RAP2 and pericentrin, PCM proteins involved in recruiting and activating gammatubulin (Fong et al., 2008, Choi et al., 2010, Delaval \& Doxsey, 2010) (Fig 3E, 3F). Both

234 CDK5RAP2 and pericentrin are present in progenitor cells with amplifying centrioles, as well as 235 migrating centrioles in immature OSNs. In dendritic knobs, CDK5RAP2 was present, while 236 pericentrin signal decreased. Taken together, our data show that groups of migrating OSN 237 centrioles remain associated with microtubule nucleation proteins and pericentriolar material, in contrast to the centrioles of many other types of mammalian neurons. This is similar to 
observations in MMCCs, where amplified centrioles were also reported to have associated PCM (Jurczyk et al., 2004; Vladar et al., 2012, Zhao et al., 2021).

Immature OSNs form a single cilium from the parental centriole.

243 We next used expansion microscopy to examine the events associated with arrival of the

244 centrioles at the dendritic knob and formation of cilia. We observed that in some OSN dendrite 245 tips with multiple centrioles, near the apical surface, a single cilium was present (Fig 4A, 4A').

246 We hypothesized that this reflected an inherent asymmetry in centriole states, such that only

247 one centriole is competent to form a cilium at this stage prior to formation of the full complement

248 of cilia. Ciliogenesis is a multi-step process that, in many organisms, requires a mature centriole

249 to bear distal and subdistal appendages. To assess distal appendage acquisition, we used expansion microscopy to image CEP164 (Centrosomal Protein 164), a distal appendage protein required for ciliogenesis (Tanos et al., 2013, Cajanek \& Nigg, 2014). In OSNs with a single cilium, distal appendages were present on the centriole at the base of the cilium and not at other centrioles (Fig 4A, 4A'), confirming that an inherent asymmetry in centriole states exists. By contrast, in OSNs with multiple cilia, many centrioles acquired distal appendages, indicating that centriole maturation and remodeling occurred at the apical surface (Fig 4B).

How might this centriole asymmetry arise? Based on our previous results regarding centriole amplification (Ching \& Stearns, 2020), we hypothesized that in progenitor cells, a single, mature, parental centriole segregates to each daughter cell whereas the newly amplified centrioles remain immature and do not acquire appendages. In support of this hypothesis, we found that both in migrating centrioles (Fig 4C, Fig 4-Supp Fig 1A, Fig 4-Supp Fig 1B) and in centrioles in the process of amplification (Fig 4-Supp Fig 1C), only a single centriole was found to have distal and subdistal appendages. These data suggest that the centriole modifications required to make cilia occur after centriole migration during OSN differentiation, and that the already-modified parental centriole is able to make a cilium before all other centrioles. The temporal progression of centriole maturation is consistent with the presence of markers of immature centrioles on some migrating centrioles (Fig 3B, 3C).

In tracheal MMCCs, the parental centriole retains its unique structural characteristics even in 270 mature cells after all cilia form (Liu et al., 2020). In particular, the parental centriole has a radial 271 array of subdistal appendages, in contrast to all other centrioles, which bear a single basal foot.

272 Radial subdistal appendages and basal feet share molecular components, but can be 
273 distinguished by the arrangement of these components around centrioles. To test whether the

274 parental centriole could be distinguished from the other centrioles in mature OSNs, we stained

275 for the shared component centriolin, using expansion microscopy. In migrating centrioles,

276 centriolin was present on only one centriole, surrounding that centriole in a subdistal

277 appendage-like pattern (Fig 4-Supp Fig 1B). In cells in which this centriole formed a single

278 cilium, centriolin retained this subdistal appendage-like pattern around the base of the cilium,

279 while other centrioles had acquired single puncta of centriolin (Fig 4D). In mature OSNs with

280 multiple cilia, one centriole was surrounded by centriolin whereas the other centrioles still had

281 only single foci of centriolin, consistent with basal feet as observed in multiciliated epithelial cells

282 (Fig 4E, Fig4-Supp Fig 1D). We conclude that in OSNs, as in multiciliated epithelial cells, the

283 cilium nucleated by the parental centriole has unique structural characteristics compared to the

284 other cilia at the mature dendritic knob.

Microtubule dynamics are necessary for centriole migration in OSNs. and transport. Neurons contain many stable microtubules as well as a population of dynamic microtubules (Baas et al., 2016). We sought to determine whether perturbation of the dynamic microtubules would affect centriole migration to the dendrite tip. To facilitate these experiments, we developed an explant culture system that retained the architecture of the epithelium in vivo. Olfactory epithelium dissected from the septa of mice expressing eGFP-centrin2 and Arl13bmCherry was plated on transwell filters. To mimic the environment that the olfactory epithelium experiences in vivo, the bottom compartment was filled with medium to create an air-liquid interface at the filter (Fig 5A).

First, we tested whether explant cultures were viable over the required time course and whether cells retained the ability to progress through the cell cycle. We found that EdU, which labels newly-replicated DNA, incorporated into nuclei near the basal lamina in the explant, indicating that cells were able to replicate DNA (Fig 5B, 5C). In addition, many of the EdU-positive cells appeared to have amplified centrioles, in accordance with our previous observations (Ching \&

302 Stearns, 2020). We treated explants with EdU for two timepoints, one and six hours post-

303 dissection, and found that the number of EdU-positive nuclei increased from 0.63 nuclei per 100 $304 \mu \mathrm{m}$ basal length of epithelium to 0.96 nuclei per $100 \mu \mathrm{m}$ of epithelium (total lengths quantitated $305=634.84 \mu \mathrm{m}$ and $621.52 \mu \mathrm{m}$ for one and six hours, respectively). Treatment during the time 306 course did not affect overall morphology of the olfactory epithelium. These results demonstrate 
that cells in the explant cultures can synthesize DNA and amplify centrioles, and that the culture retains its characteristic architecture during the time course.

Next, we used this explant culture system to determine whether microtubule dynamics are required for centriole migration. Explant cultures were treated for one hour or six hours with paclitaxel (taxol), a microtubule stabilizing drug, or DMSO as a vehicle control. Treatment with

313 DMSO or paclitaxel for one hour or six hours did not affect overall tissue morphology, including 314 the actin-rich apical domain (Fig 5D, 5E). We then scored the number of migrating centriole 315 groups in distinct regions of the olfactory epithelium: the subapical region, and the middle and 316 basal region (regions marked in Fig 1A). In all conditions, more centriole groups were found in 317 the subapical compartment than in the middle and basal region, consistent with previous reports (Mulvaney \& Heist, 1971). Upon treatment with paclitaxel, more centrioles were found in the subapical compartment (Fig 5F). After one hour of paclitaxel treatment, the subapical compartment had 1.38-fold more centrioles than control (total basal lengths quantitated = 905.37 $\mu \mathrm{m}$ and $988.17 \mu \mathrm{m}$ for paclitaxel and DMSO, respectively). Strikingly, after six hours of paclitaxel treatment, the subapical compartment had 2.58-fold more centrioles compared to control (total basal lengths quantitated $=1019.76 \mu \mathrm{m}$ and $1126.44 \mu \mathrm{m}$ for paclitaxel and DMSO, respectively, Fig 5F). The subapical accumulation of centrioles with paclitaxel treatment suggests that microtubule dynamicity is required for centriole groups to complete their progression to the apical membrane.

\section{Discussion}

Olfactory sensory neurons (OSNs) in vertebrates have a morphology specialized for their role, with an array of sensory cilia at the tip of a dendrite. We showed previously that the centrioles required to form these cilia are produced in progenitor cells that undergo centriole amplification and divide before becoming OSNs (Ching \& Stearns, 2020). Here we have examined centriole migration and maturation in OSNs, with a focus on the unique biology of OSNs. We established new techniques for manipulating and studying the olfactory epithelium, applying expansion microscopy, live imaging, and an explant system. These will be useful for future experiments on

338 the cells of this tissue, important for their sensory role and as a point of entry for respiratory 339 infection. 
341 Our results are summarized in Figure 6. Progenitor cells, located near the basal lamina of the

342 olfactory epithelium, amplify centrioles from an initial pair. The progenitor cell divides and the

343 daughter cell differentiates into an immature OSN, which extends a dendrite towards the apical

344 surface. Centrioles migrate in multiple groups within a single dendrite in immature OSNs. The

345 leading group of centrioles moves in tandem with dendrite extension and can be followed by

346 lagging groups. Centriole groups move relatively slowly towards the apical end of the dendrite

347 and during migration most of the centrioles retain markers associated with immature centrioles

348 and lack the appendages required to make cilia. The exception is a single migrating centriole,

349 presumably an original parental centriole, which has appendages and is able to form a single

350 cilium in the dendritic knob, prior to the others. Finally, most centrioles acquire distal and

351 subdistal appendages and form the dozens of olfactory cilia found in the mature OSN. The

352 parental centriole retains structural characteristics that differentiate it from the other centrioles

353 within the same cell.

We previously showed that centrioles in OSNs are amplified, at least in part, by formation of centriole rosettes in progenitor cells, without deuterosomes like those seen in MMCCs (Ching \& Stearns, 2020). Here we exploited the resolution afforded by expansion microscopy to precisely count centrioles, finding that the number of centrioles during migration and in mature OSNs was even higher than previously reported, with a mean of approximately 36 centrioles in the latter. The number of centrioles was similar for both states, suggesting that all centriole amplification takes place prior to the initiation of migration and dendrite extension. This is a higher number of centrioles than reported by Uytingco et al. (2019) and Ukhanov et al. (2022); this could be due to differences in microscopy and counting methods, to regional differences in the mouse olfactory epithelium (Challis et al., 2015), or to variation between animals. Based on the new evidence we present here, a parsimonious explanation for how OSNs achieve their final centriole number is that amplification is initiated by rosette formation around the two parental centrioles in progenitor cells, followed by a single mitotic division and centriole disengagement from the rosettes, and further amplification in post-mitotic cells that become OSNs.

OSNs are, to our knowledge, unique in that they undergo a mitotic division with amplified

371 centrioles, which is then followed by further amplification (Ching \& Stearns, 2020). Thus, an

372 immature OSN potentially has centrioles of three different ages: the parental centriole, amplified 373 centrioles formed prior to mitosis, and those that may have formed after mitosis. We found that 374 some of the amplified centrioles in immature OSNs have nucleation-associated proteins of the 
375 PCM, including gamma-tubulin, CDK5RAP2, and pericentrin, consistent with a portion of the

376 amplified centrioles having undergone the centriole-to-centrosome conversion as described in

377 cultured mammalian cells (Wang et al., 2011; Izquierdo et al., 2014). Similarly, some of the

378 centrioles in immature OSNs have the cartwheel components SASS6 and STIL, as might be

379 expected for those centrioles formed after the mitotic division, whereas all centrioles lack these

380 components in mature OSNs.

382 Centrioles in mature OSNs have the proteins associated with rootlets, distal appendages and

383 subdistal appendages/basal feet, typical of centrioles underlying cilia in other multiciliated cells

384 (Anderson \& Brenner 1971; Menco et al., 1978; McClintock et al., 2008; Sorokin 1968). It is

385 interesting that the final modification of the non-parental centrioles with these components in

386 OSNs appears to be controlled spatially, by their arrival in the forming dendritic knob, as they

387 were absent on migrating centrioles in immature OSNs. This might occur, for example, by

388 localized translation or accumulation of the protein components of these structures or the

389 regulators of their assembly on the centriole. In cycling mammalian cells grown in culture,

390 centriole maturation is driven by cell cycle progression and the activity of PLK1 kinase (Kong et

391 al., 2014, Shukla et al., 2015). In MMCCs, low-level activation of mitotic kinases is important for

392 centriole amplification (Al Jord et al., 2017). In OSNs, it is possible that centriole maturation is

393 also controlled by PLK1 and other mitotic regulators, with the added element of spatial control.

394 Interestingly, it has also been shown that loss of centrin2 results in fewer cilia on OSNs as well

395 as more OSNs with centrioles below the apical surface (Ying et al., 2014). This would be

396 consistent with a defect in acquisition of appendages, although the mechanism by which

397 centrin2 affects centriole docking is unclear.

The presumptive parental centriole differed from all others in OSNs in having subdistal and distal appendage proteins prior to its migration to the dendrite tip. This was apparent during

401 migration of centrioles and in the early stage of cilium formation when only this centriole was

402 associated with a cilium. The presence of a single cilium before formation of the full complement

403 of multiple cilia might enable signaling important for OSN differentiation, as has been proposed

404 for the primary cilium of MMCCs that precedes multiciliated differentiation (Jain et al., 2010),

405 although we note that Schwarzenbacher, et al. (2005) found that the earliest OSN cilia formed

406 lack olfactory receptors. The parental centriole retained a radial array of the subdistal

407 appendage component centriolin even in the mature OSN, whereas all other centrioles had a

408 single focus of centriolin, consistent with them having basal feet. This is remarkably similar to 
the situation in MMCCs in which a single parental centriole also differed from other centrioles and was associated with what was termed a "hybrid cilium" (Liu et al., 2020). It is unclear what prevents the parental centriole from forming a cilium prior to reaching the dendritic knob, although it might be due to the same spatial regulation that we postulated to control centriole maturation.

How do centrioles migrate from the cell body to the tip of the dendrite? Our work rules out several possibilities. First, centrioles within a single cell often travel in multiple groups separated by relatively long distances along the dendrite. This excludes the possibility that forces act upon only a single centriole, for instance the parental centriole. Second, our data also exclude the possibility that centrioles might migrate by docking to membranes, as only the presumptive parental centriole bears the distal appendages required for membrane association during migration. Third, migrating centrioles lack rootletin, ruling out models in which centrioles are moved by attachment of centriolar rootlets to actin filaments, as observed during macrociliary cell development in the comb jelly Beroe (Tamm \& Tamm, 1988).

Our data support a model in which the microtubule cytoskeleton is critical for centriole migration. Using a novel explant system, we found that inhibiting microtubule dynamics disrupts centriole migration to the apical surface. In this respect, OSNs are similar to MMCCs of the oviduct, in which paclitaxel-mediated stabilization of microtubules does not affect centriole biogenesis, but can inhibit centriole migration (Lemullois et al., 1988; Boisvieux-Ulrich et al., 1989). Because of the very different cell and tissue architectures in MMCCs compared to OSNs it is unclear whether the mechanism of inhibition by microtubule stabilization is the same in these cell types.

432 Our establishment of an explant system will be useful for future work in distinguishing the exact

433 mechanisms by which cytoskeletal elements influence centriole migration in OSNs.

How do forces act upon migrating centrioles? One possibility is that microtubule motors are

436 directly involved in centriole transport. In the C. elegans ciliated PQR neuron, SAS-5, an

437 orthologue of STIL, directly interacts with a dynein light chain (DLC-1/LC8), suggesting that

438 centrioles may be cargos of cytoplasmic dynein in the dendrite (Li et al., 2017). In addition, the

439 rate of migration for the single centriole that migrates in C. elegans neurons is similar to that of 440 mouse OSN centrioles (in OSNs: maximum of $0.18 \mu \mathrm{m} / \mathrm{min}$; in C. elegans: $0.076 \mu \mathrm{m} / \mathrm{min}$, Li et 441 al., 2017), suggesting that similar mechanisms may drive centriole migration in both contexts.

442 However, dynein motors acting processively exhibit velocities much higher than those observed 
443 in OSNs or the PQR neuron, approximately $60 \mu \mathrm{m}$ per minute in cells and in vitro (Presley et al.,

444 1997; King \& Schroer, 1999). Thus, dynein might act non-processively and/or interact with

445 centrioles transiently in OSNs. This is reminiscent of slow axonal transport, which drives the

446 anterograde transport of cytoskeletal elements within neuronal axons (Baas \& Buster, 2004;

447 Roy, 2020). Recent work has indicated that cargos undergoing slow axonal transport interact

448 transiently or intermittently with molecular motors (Roy, 2020), and it is possible that similarly

449 transient interactions are responsible for centriole movement in OSN dendrites.

Our finding that microtubule nucleation proteins are present on migrating centrioles suggests

452 another, non-mutually exclusive hypothesis: that migrating centrioles are microtubule organizing

453 centers, with self-nucleated microtubules involved in their motility. Force could be derived from

454 microtubule polymerization itself, or by interaction of nucleated microtubules with an existing

455 dendritic microtubule array. In other mammalian neurons, centrioles remain near the nucleus

456 and lose microtubule nucleating proteins as the cell differentiates (Leask et al., 1997; Steiss et

457 al., 2010; Yonezawa et al., 2015; Sanchez-Huertas et al., 2016; Wilkes \& Moore, 2020). In

458 cycling mammalian cells, PCM nucleates microtubule asters that center and position the

459 centrosome. This occurs through pushing forces driven by microtubule nucleation, and pushing

460 and pulling forces driven by molecular motors (reviewed in Burakov \& Nadezhdina, 2020).

461 Similarly, microtubule nucleation may help orient and position centrioles toward the apical

462 surface in OSNs. This would be consistent with a model in which forces are acting upon multiple

463 centrioles, perhaps even individual centrioles, within the same dendrite.

In addition to migration within dendrites, the PCM of OSN centrioles might also influence dendrite microtubule organization itself. OSN dendrites have a polarized microtubule

467 cytoskeleton, in which the microtubule minus ends are oriented out, toward the dendritic tips

468 (Burton, 1985). This polarity, observed in bullfrog OSNs, is in contrast to dendrites of many

469 neurons, which have a mixed polarity. An attractive possibility is that the centrioles positioned at

470 the tip of the dendrite function as a centrosome, nucleating microtubules, with their minus ends

471 out. Supporting this hypothesis, we observed that PCM proteins are present at centrioles in both

472 immature and mature OSNs. An interesting parallel is that microtubule polarity in dendrites of

473 non-ciliated C. elegans PVD neurons is established by a non-centrosomal microtubule

474 organizing center that is present at the dendritic tip (Liang et al., 2020), suggesting that this may

475 be a conserved mechanism of establishing and maintaining microtubule polarity in dendrites. 
477 Finally, we have focused here on microtubule-based mechanisms, but acknowledge that other

478 mechanisms, for example based on the actin cytoskeleton, might also be important for centriole

479 migration and docking in OSN dendrites. We expect that the new techniques for manipulating

480 and studying the olfactory epithelium that we describe will facilitate further research into these

481 and other questions about how multiple centrioles and cilia are formed in OSNs and participate

482 in their structure and function.

\section{Methods}

\section{Ethics Statement}

488 This study uses samples from mice. All animal procedures in this study were approved by the 489 Stanford University Administrative Panel for Laboratory Animal Care (SUAPLAC protocol 11659) and carried out according to SUAPLAC guidelines.

Mice

493 Olfactory epithelium samples were taken from both male and female mice, euthanized by $\mathrm{CO}_{2}$

494 inhalation, for all experiments. Mice were between 1 and 6 months old. (See each section for 495 specific ages.) Mice constitutively overexpress eGFP-centrin2 to mark centrioles, and Arl13b496 mCherry to mark primary cilia (line generated by Bangs et al., 2015). In no instance did we 497 observe Arl13b-mCherry in olfactory cilia, but Arl13b-mCherry was present in primary cilia of 498 olfactory epithelium progenitor cells. Native fluorescence signal from eGFP and mCherry was 499 not preserved in expansion microscopy.

501 Microscopy

502 All confocal microscopy images were acquired as single planes or Z-stacks collected at $0.27-\mu \mathrm{m}$ 503 intervals using a Zeiss Axio Observer microscope (Carl Zeiss) with a PlanApoChromat $63 \times / 1.4$ 504 NA objective, a Yokogawa CSU-W1 head, and a Photometrics Prime BSI express CMOS 
camera. Slidebook software (Intelligent Imaging Innovations, 3i) was used to control the

506 microscope system.

507

\section{$508 \quad$ Antibodies}

509 Primary antibodies used for immunofluorescent staining are listed in Table 1. AlexaFluor-

510 conjugated secondary antibodies (Thermo-Fisher) were diluted 1:1000 for standard

511 immunofluorescence and 1:500 or 1:1000 for expansion microscopy.

513 Table 1. Antibodies used in this study.

\begin{tabular}{|c|c|c|}
\hline target & source & dilution \\
\hline acetylated tubulin & $\begin{array}{l}\text { Sigma-Aldrich, clone 6-11B-1, } \\
\text { RRID:AB_477585 }\end{array}$ & $1: 1000$ \\
\hline CDK5RAP2 & $\begin{array}{l}\text { Millipore, 06-1398 rabbit polyclonal, } \\
\text { RRID:AB_11203651 }\end{array}$ & $1: 200$ \\
\hline centrin & $\begin{array}{l}\text { EMD Millipore, clone } 20 \mathrm{H} 5 \\
\text { RRID:AB_10563501 }\end{array}$ & $1: 1000$ \\
\hline CEP164 & $\begin{array}{l}\text { rabbit polyclonal previously described in Lee } \\
\text { et al } 2014\end{array}$ & $1: 500$ \\
\hline GFP & $\begin{array}{l}\text { rabbit antibody previously described in } \\
\text { Hatch et al., } 2010\end{array}$ & $1: 2000$ \\
\hline GAP43 & Abcam, clone EP890Y, RRID:AB_1310252 & $\begin{array}{l}1: 750 \text { (requires } \\
\text { overnight, } 4-^{\circ} \mathrm{C} \\
\text { incubation) }\end{array}$ \\
\hline Pericentrin & $\begin{array}{l}\text { BD Biosciencies, clone 30, } \\
\text { RRID:AB_399294 }\end{array}$ & $1: 500$ \\
\hline Centriolin & $\begin{array}{l}\text { Santa Cruz Biotech, clone C-9, RRID: } \\
\text { AB_10851483 }\end{array}$ & $1: 50$ \\
\hline STIL & $\begin{array}{l}\text { Abcam, rabbit polyclonal, } \\
\text { RRID:AB_2197878 }\end{array}$ & $1: 1000$ \\
\hline
\end{tabular}




\begin{tabular}{|l|l|l|} 
SASS6 & Santa Cruz Biotech, RRID:AB_1128357 & $1: 200$ \\
\hline ODF2 & $\begin{array}{l}\text { Novus Biologicals, Mouse IgG2a, } \\
\text { RRID:AB_1146453 }\end{array}$ & $1: 200$ \\
\hline rootletin (CROCC) & $\begin{array}{l}\text { Santa Cruz Biotech, clone C-2, } \\
\text { RRID:AB_10918081 }\end{array}$ & $1: 100-200$ \\
\hline B-tubulin III & BioLegend, clone TuJ1, RRID:AB_2313773 & $1: 1000-2000$ \\
\hline gamma-tubulin & $\begin{array}{l}\text { Sigma-Aldrich, clone GTU88, } \\
\text { RRID:AB_532292 }\end{array}$ & $1: 1000$ \\
\hline
\end{tabular}

Immunofluorescent staining of cryosections

517 Olfactory epithelia were dissected mechanically from mice that were 1 to 4 months old. All

518 stainings were performed at least twice, in olfactory epithelium taken from both a male and a

519 female mouse. After initial snout removal, fine dissection was performed in a dish of cold

520 Tyrode's solution (140mM NaCl, $5 \mathrm{mM} \mathrm{KCl}, 10 \mathrm{mM} \mathrm{HEPES,} 1 \mathrm{mM} \mathrm{CaCl}_{2}, 1 \mathrm{mM} \mathrm{MgCl}_{2}, 1 \mathrm{mM}$

521 sodium pyruvate, $10 \mathrm{mM}$ glucose in $\mathrm{ddH}_{2} \mathrm{O}$ ), as in other reports (Oberland and Neuhaus, 2014)

522 and at github.com/katieching/Protocols. Whole olfactory epithelia, turbinate epithelia, or septum

523 epithelia were fixed immediately in $4 \%$ PFA in PBS at $4^{\circ} \mathrm{C}$ for 3 to 24 hours. Samples were then

524 washed in phosphate buffered saline (PBS) and stored at $4^{\circ} \mathrm{C}$. Before mounting, samples were

525 equilibrated in 1 to $5 \mathrm{~mL}$ of $30 \%$ sucrose solution in water for a minimum of 12 hours at $4^{\circ} \mathrm{C}$.

526 Samples were embedded in OCT compound (Sakura Tissue-Tek) on dry ice and stored at -

$52780^{\circ} \mathrm{C}$. Embedded samples were sectioned at $14 \mu \mathrm{m}$ on a Leica cryostat and adhered to charged

528 slides by drying at room temperature for approximately 1 hour. Slides were stored with drying

529 pearls (Thermo Fisher) at $-80^{\circ} \mathrm{C}$ and thawed under desiccation no more than twice. Sections

530 were outlined with a hydrophobic pen. Residual free aldehydes were quenched while samples

531 were rehydrated in PBS with $0.3 \mathrm{M}$ glycine, $1 \%$ calf serum, and $0.1 \%$ Triton-x 100 for 0.5 hours

532 at room temperature. Samples were blocked and permeabilized for an additional 0.5 to 4 hours 
533 in PBS with $1 \%$ calf serum and $0.1 \%$ Triton-x 100. Antibodies were also diluted in PBS with $1 \%$

534 calf serum and $0.1 \%$ Triton-x 100 (see Table 1). Slides were incubated with primary antibodies

535 for approximately 3 hours at room temperature or overnight at $4^{\circ} \mathrm{C}$, washed in PBS, incubated

536 with secondary antibodies for approximately 1 hour at room temperature, washed in PBS,

537 incubated in DAPI diluted 1:1000 for 5 minutes, washed in PBS, and mounted in MOWIOL.

538 Where phalloidin conjugated to Alexa Fluor 568 was used, it was included at the secondary

539 antibody step at a 1:1000 dilution (Molecular Probes). Where EdU was used, incorporation was

540 visualized using the Click-iT Alexa Fluor 594 kit according to the manufacturer's instructions

541 (Molecular Probes). Slides were imaged by spinning disk confocal microscopy, and images

542 were acquired with the SlideBook software by Intelligent Imaging Innovations (3i). Images were

543 processed in FIJI (Schindelin et al., 2012). Images are rotated to orient the apical surface

544 toward the top of the page. For images with high background, contrast in the representative

545 images was adjusted uniformly across the image such that the area outside of cells was black

546 and areas of high signal were just below saturation.

Expansion microscopy - olfactory epithelium

549 Details about expansion microscopy for olfactory epithelium can be found at

550 github.com/katieching/Protocols and were based on work by Gambarotto et al., 2019 and

551 Sahabandu et al., 2019. In brief, samples from 1- to 4-month-old mice were dissected

552 mechanically in a dish of cold Tyrode's solution (140mM NaCl, $5 \mathrm{mM} \mathrm{KCl}, 10 \mathrm{mM} \mathrm{HEPES}, 1 \mathrm{mM}$

$553 \mathrm{CaCl}_{2}, 1 \mathrm{mM} \mathrm{MgCl} 2,1 \mathrm{mM}$ sodium pyruvate, $10 \mathrm{mM}$ glucose in $\mathrm{ddH}_{2} \mathrm{O}$ ), fixed, washed, and

554 cryoprotected by the same methods used for unexpanded immunofluorescent staining. All

555 stainings were performed at least twice, in olfactory epithelium taken from both a male and a

556 female mouse. Samples were embedded in OCT (Sakura Tissue-Tek) on dry ice and stored at -

$55780^{\circ} \mathrm{C}$. Embedded samples were sectioned at $14 \mu \mathrm{m}$ thickness on a Leica cryostat, placed on

558 charged glass slides within a border pre-drawn with hydrophobic pen, and allowed to thaw for 1 
559 minute. Sections were then washed three times in PBS for approximately 5 minutes per wash.

560 PBS was removed, and sections were incubated in a monomer fixative solution $(0.7 \%$

561 formaldehyde and $1 \% \mathrm{w} / \mathrm{v}$ acrylamide in water) for 2 hours at room temperature. Sections were

562 washed once in monomer solution ( $19 \% \mathrm{w} / \mathrm{v}$ sodium acrylate, $10 \% \mathrm{w} / \mathrm{v}$ acrylamide, and $0.1 \%$

563 BIS in PBS). Solution was removed, and slides containing sections were inverted onto droplets

564 of cold gelation solution (monomer solution with TEMED and ammonium persulfate added to a

565 final concentration of $0.5 \%$ each). Stacks of approximately five coverslips were used as spacers

566 to achieve the desired gel thickness. Gels were set for 5 minutes on ice and then overnight at

567 room temperature in a sealed chamber with wet paper towels. Slides with gels attached were

568 incubated at $95^{\circ} \mathrm{C}$ in a large volume of denaturation buffer (200 mM SDS, $200 \mathrm{mM} \mathrm{NaCl}$, and 50

$569 \mathrm{mM}$ Tris in water) for approximately 4 to 6 hours. Gels were washed and then pre-expanded in

570 water overnight. Gels were then incubated in PBS for at least 30 minutes. Gels were incubated

571 in primary antibody solution in PBS with or without $3 \%$ bovine serum albumin and $0.1 \%$ Triton-

$572 \times 100$ (see Table 1) overnight on a nutator at $4^{\circ} \mathrm{C}$. Gels were washed three times in PBS for 10

573 to 30 minutes per wash and then incubated in a solution of secondary antibodies conjugated to

574 Alexa Fluors plus DAPI, diluted 1:500 or 1:1000, overnight with gentle agitation at $4^{\circ} \mathrm{C}$. Gels

575 were washed in PBS for at least 30 minutes, then in water three times for 30 minutes per wash.

576 Gels were then allowed to fully expand in water for at least an hour before mounting in a glass-

577 bottom imaging dish, and imaging by spinning disk confocal microscopy. Native fluorescence

578 signal from eGFP and mCherry was not preserved. As needed, gels were imaged in a poly-

579 lysine-coated dish to reduce sample movement during acquisition. Centriole counts were semi-

580 automated: an initial volume segmentation was performed in Imaris, followed by manual

581 inspection. Graphs for Fig 1 - Supp 1 were made using GraphPad Prism.

583 Live imaging of olfactory epithelium 
584 Septal or flattened turbinate olfactory epithelium was mechanically dissected from 3.5- to 6-

585 month-old mice in the same manner as for explant preparation. Imaging was performed in

586 samples taken from three different animals and included both male and female mice. The

587 procedure for mounting was partly based on work from Williams et al. (2014). For this study,

588 dissected samples were pulled onto a coverslip containing an agarose pad made of $1 \%$ low-

589 melt agarose (Lonza \#50100) dissolved in Tyrode's buffer. The coverslip with the sample was

590 then inverted into the center of an imaging dish containing $150 \mu \mathrm{L}$ of imaging buffer (Tyrode's

591 with $5 \%$ cosmic calf serum and $5 \mu \mathrm{g} / \mathrm{mL}$ Hoechst 34580 (Invitrogen \#H21486), mixed by pipette

592 and vortexed) and allowed to sit for at least 5 minutes before imaging. Coverslips were sealed

593 into place with melted Vaseline as needed. Samples were imaged en face by spinning disk

594 confocal microscopy at approximately $37^{\circ} \mathrm{C}$ with low laser power until no more than 4 hours

595 after euthanasia. Files were acquired and scored using the SlideBook software by Intelligent

596 Imaging Innovations (3i). For scoring, centriole groups below the apical surface were identified

597 in a 4D volume view and measured in a maximum intensity projection over the $y$ axis with

598 respect to a group of nearby mature OSN centrioles as a fiducial mark. Locations for each group

599 were scored in the first and last frame during which they were visible in the maximum projection,

600 and average rates were calculated from the difference between those measurements.

601 Kymograph analysis was performed using Dynamic Kymograph in FIJI (Zhou et al., 2020).

602

603 Culture and analysis of septum explants grown on air-liquid interface

604 Details about growing olfactory epithelium explants can be found at

605 github.com/katieching/Protocols and is partly based on the procedure described by Oberland

606 and Neuhaus (2014). In brief, septum epithelia from 1- to 4-month-old mice were dissected

607 mechanically in cold Tyrode's buffer and placed apical side up on transwell polycarbonate filters

608 with $0.4 \mu \mathrm{m}$ pore size with the bottom compartment containing additional Tyrode's buffer. All

609 experiments were performed in explants taken from both male and female mice. Once all 
610 samples were placed, buffer was aspirated from the bottom compartment and replaced with

611 warm explant medium (15\% fetal bovine serum and $0.02 \%$ L-ascorbic acid in Waymouth's

612 media (Thermo Fisher Scientific \#11220035)) (based on Farbman, 1977). No medium was

613 added to the top compartment, preserving the air-liquid interface. Relevant treatments $(10 \mu \mathrm{M}$

614 EdU (Thermo Fisher Scientific \#A10044), $15 \mu \mathrm{M}$ paclitaxel (EMD Millipore \#580555), or an

615 equivalent volume of DMSO) were mixed into media by pipetting and vortexing before addition.

616 Samples were incubated in a $33^{\circ} \mathrm{C}, 5 \% \mathrm{CO}_{2}$, humidified incubator for 1 hour or 6 hours before

617 forceps were used to transfer them from the filters to 4\% PFA in PBS for overnight fixation. For

618 Fig 3B, 3C, samples within each replicate were obtained from the same animal. For Fig 3D-F,

619 samples were matched such that explants taken from the two conditions came from the same

620 animal at the same time point. Where explants were taken from the same animal, conditions

621 were randomized between the right and left halves of the septum. Samples were then washed

622 and processed by the same method of immunofluorescent staining described above. To score

623 explants treated with paclitaxel (Fig 3F), sample size was selected by (1) measuring the

624 average length between areas of high amplification (approximately $250 \mu \mathrm{m}$ ), (2) scoring a length

625 greater than that for the condition with the most limited sample, and (3) scoring progressively

626 (i.e. not selecting regions) along the epithelium of all other conditions until the length was

627 matched. This was then repeated in samples from the opposite sex.

\section{Acknowledgements}

631 This project was supported by the National Science Foundation Graduate Research Fellowship

632 under Grant No. D-G16E5 6518 (to K.C.), and by the NIGMS of the National Institutes of Health

633 under award numbers 1R35GM130286 (to T.S.), K99 GM131024 (to J.T.W.), and

634 T32GM007276 (CMB Training Grant). We thank members of the Stearns lab for helpful

635 feedback and suggestions. 


\section{Competing interests}

639 The authors declare that no competing interests exist.

640

641

\section{Author contributions}

643 Conceptualization and writing: K.C. (ORCiD: 0000-0002-0517-2421), J.T.W. (ORCiD: 0000-

644 0002-8506-5182), and T.S. (ORCiD: 0000-0002-0671-6582); Investigation: K.C. and J.T.W.;

645 Supervision, funding acquisition, and project management: T.S.

646

647

648 
Figure 1. Centrioles in olfactory sensory neurons (OSNs) migrate tens of micrometers to

\section{2 the apical surface.}

653 (A) Overview of the olfactory epithelium. Single plane fluorescence image of a side view of the 654 olfactory epithelium (left), corresponding to a schematic of cell types in the olfactory epithelium (right). Yellow: eGFP-centrin2. Cyan: staining for acetylated tubulin, strongly marking olfactory cilia and, faintly, neuronal microtubules. Magenta: staining for $\beta$-tubulin III, marking neuronal microtubules. White: DAPI, marking DNA. The apical surface is oriented at the top of this image and all subsequent side view images. Dashed line: apical surface.

(B) Mature olfactory sensory neurons. Single plane, en face fluorescence image of the apical Double solid line: basal lamina. Boxes show the relative positions of critical stages of OSN differentiation: (i) The subapical compartment of the olfactory epithelium, defined as the space between the bottom of the sustentacular cell nuclei and the apical surface with olfactory cilia. (ii) A group of centrioles migrating through the middle of the olfactory epithelium, below the sustentacular cell nuclei. (iii) A progenitor cell near the basal lamina.

\section{2} surface of the olfactory epithelium. Green: eGFP-centrin2. Magenta: dye-conjugated Scale bar $=10 \mu \mathrm{m}$.

\section{3} phalloidin, marking an enrichment of F-actin at the apical borders of sustentacular cells. Scale bar $=10 \mu \mathrm{m}$.

(C) Schematic of Fig 1B depicting a sustentacular cell wrapping around the dendrites of nearby OSNs. Green: OSN dendrite. Gray: sustentacular cell cytoplasm. Magenta: F-actin. Wavy black lines: multiple OSN cilia.

(D) Centriole migration in the olfactory epithelium. Single plane fluorescence image of a side view of the subapical compartment of the olfactory epithelium. Green:eGFP-centrin2. 

$\mu \mathrm{m}$.

676 (E) Mature olfactory sensory neurons, as imaged by expansion microscopy. Single plane

677 fluorescence image of the en face apical surface. White: staining for acetylated tubulin, 678 marking centrioles, cilia, and, faintly, neuronal microtubules. Multiple cilia can be seen 679 protruding from mature OSNs. A green arrow marks a dendrite with a group of migrating 680 centrioles arriving at the apical tip. Scale bar $=2 \mu \mathrm{m}$.

681 (F) Centriole migration, as imaged by expansion microscopy. Single plane fluorescence image of a side view of the subapical compartment. White: staining for acetylated tubulin, marking centrioles, cilia, and, faintly, neuronal microtubules. Cilia can be seen at the apical surface, and green arrows mark groups of migrating centrioles. Scale bar $=2 \mu \mathrm{m}$.

Figure 1-figure supplement 1. Centriole and cilium numbers in OSN progenitors and mature OSNs. (A) Example of Imaris segmentation of centrioles in progenitor cells. (i) 3D image of centrioles in a progenitor cell. Green: staining for acetylated tubulin. (ii) The same image after segmentation in Imaris. Segmented objects are color-coded with a LUT to encode zdepth. After automated counting, each image was visually inspected, and counts were corrected where objects and centrioles or cilia did not correspond. Scale bar $=2 \mu \mathrm{m}$. (B) Example of Imaris segmentation of centrioles and cilia in mature OSNs. (i) 3D image of a mature OSN dendritic knob. Green: staining for acetylated tubulin. (ii) The same image after segmentation in Imaris. Segmented objects are color-coded with a LUT to encode z-depth. After automated counting, each image was visually inspected, and counts were corrected where objects and centrioles or cilia did not correspond. Scale bar $=2 \mu \mathrm{m}$.

(C) Plot of the number of centrioles per cell in progenitor cells and mature OSNs. Red dots: sample 1, three-month-old female. Black dots: sample 2, one-month-old male. Mean and 
standard error of the mean (SEM) are graphed. In progenitors: mean $=26.40$ centrioles, $\mathrm{SEM}=2.234$ and standard deviation $=9.992$. In mature OSNs: mean $=36.46$ centrioles, $\mathrm{SEM}=1.665$, and standard deviation $=8.156$

(D) Plot of centriole and cilium numbers in mature OSNs. Black dots: percentage of centrioles that have cilia. Purple squares: number of cilia in each cell. Each dot and square in the same column belong to the same dendritic knob. Measurements were arranged along the $\mathrm{x}$-axis in order of increasing percentage of centrioles with cilia. Mean: $85 \%$ of centrioles at a dendritic knob nucleated cilia $(S E M=2.47$; standard deviation $=12.10)$. Mean cilia number per dendritic knob: 30.54 cilia (SEM=1.455; standard deviation $=7.126)$.

Figure 2. Centrioles migrate in multiple groups during dendrite elongation.

711 (A) Within a single dendrite, centrioles migrate in multiple groups. Expansion microscopy - side view of expanded olfactory epithelium. In all side-view images, apical is oriented toward the top of the image. White: staining for acetylated tubulin. Maximum z-projection of confocal stack. Mature OSNs have multiple cilia, which are visible at the apical surface. Green arrows: groups of centrioles migrating separately within the dendrite of a single OSN. Scale bar $=2 \mu \mathrm{m}$.

(B) Centriole migration occurs concomitantly with dendrite elongation. Fluorescence image of a side view of the olfactory epithelium. The leftmost image is a maximum intensity projection, and the right panels show a single plane. Cyan: staining for GAP43. Magenta: staining for $\beta$ -

(C) A centriole group migrates toward the apical surface. i) Live time lapse imaging of olfactory epithelium. Maximum x-projection image, showing a side view. White: eGFP-centrin2. Blue: Hoechst, marking DNA in the most apical layer of nuclei, which are mostly sustentacular cells. Green arrows: a group of centrioles moving toward the apical surface at 0.18 

labels indicate orientation of the sample in the kymograph.

(D) A centriole group with no net movement. i) Live time lapse imaging of olfactory epithelium, highlighting a different centriole group from the same acquisition as Fig 2C. Maximum xprojection image, showing a side view. White: eGFP-centrin2. Blue: Hoechst. Green arrows: a group of centrioles that have no net movement. See Figure 2 - Source Data 1 for migration rates. ii) Kymograph illustrating a lack of total migration of the centriole group. Apical and basal direction labels indicate orientation of the sample in the kymograph.

Figure 2-figure supplement 1. A centriole group migrates toward the apical surface. nuclei, which are mostly sustentacular cells. Time between frames $=2$ minutes.

Figure 2-figure supplement 2. A centriole group with no net movement.

742 Movie of the same centriole group as depicted in Fig 2D. Maximum x-projection image, showing 743 a side view. White: eGFP-centrin2. Blue: Hoechst. Time between frames $=2$ minutes.

Figure 3. Composition of centriole groups during OSN differentiation.

746 (A) The cohesion fiber and striated rootlet protein rootletin is absent during centriole migration

747 but is gained at the mature dendritic knob. Expansion microscopy, maximum z-projection of 748 confocal stack. In all side-view images, apical is oriented toward the top of the image. Cyan: 749 staining for Rootletin (CROCC). Magenta: staining for acetylated tubulin. Yellow: staining for

750 centrin. (i) Side view of migrating centrioles. Centrioles below the apical surface, in the 
dendrite of an immature OSN. (ii) Centrioles at the apical surface in the same sample as those shown in Fig 3Ai. Scale bars $=2 \mu \mathrm{m}$.

(B-F) Expansion microscopy - Single plane fluorescence images of centrioles in progenitor cells

754 (left column), immature OSNs with migrating centrioles (middle column, white lines outline an

755 OSN dendrite), and mature OSNs imaged en face (right column). Scale bars $=2 \mu \mathrm{m}$.

756 (B) The immature centriole protein STIL is present in progenitors and immature OSNs. Green:

757 staining for STIL. Magenta: staining for acetylated tubulin.

758 (C) The immature centriole protein SASS6 is present in progenitors and immature OSNs.

759 Green: staining for SASS6. Magenta: staining for centrin.

760 (D) The pericentriolar material protein gamma-tubulin is present throughout OSN differentiation.

761 Green: staining for gamma-tubulin. Magenta: staining for acetylated tubulin.

762 (E) The pericentriolar material protein CDK5RAP2 is present throughout OSN differentiation.

763 Green: staining for CDK5RAP2. Magenta: staining for acetylated tubulin.

764 (F) The pericentriolar material protein pericentrin (PCNT) is present in progenitors and immature OSNs. Green: staining for pericentrin (PCNT). Magenta: staining for acetylated tubulin.

Figure 4. A single cilium forms prior to formation of multiple cilia.

768 (A) An OSN bearing a single cilium in the subapical compartment of the olfactory epithelium.

769 Expansion microscopy - single plane fluorescence image of a side view of the subapical

770 compartment. In all side-view images, apical is oriented toward the top of the image. Cyan:

771 staining for CEP164, marking the location of distal appendages. Magenta: staining for

772 acetylated tubulin, marking centrioles, cilia, and, faintly, neuron microtubules. Yellow:

773 staining for centrin, marking centrioles. White: DAPI, marking DNA. A box marks the location

774 of the inset, $A^{\prime}$. Scale bar $=10 \mu \mathrm{m}$. (A') Immature OSN with a single cilium, inset from panel

775 A. Magenta: staining for acetylated tubulin. Yellow: staining for centrin. Cyan: staining for

776 CEP164, marking distal appendages at the base of the cilium. Arrow: Cep164 is only found 
at the base of the cilium, and not present on other centrioles within the dendrite. Scale bar $=$

778 $2 \mu \mathrm{m}$.

779

(B) During centriole migration in immature OSNs, a single centriole bears Cep164. Expansion microscopy - single plane fluorescence image of migrating centrioles in a side view of the olfactory epithelium. Green: staining for CEP164. Magenta: staining for acetylated tubulin. Scale bar $=2 \mu \mathrm{m}$.

(C) In mature OSNs, multiple cilia bear Cep164. Expansion microscopy - single plane fluorescence image of a mature OSN with multiple cilia. Green: staining for CEP164, marking distal appendages at the base of cilia. Magenta: staining for acetylated tubulin. Scale bar $=2 \mu \mathrm{m}$.

(D) In OSNs bearing a single cilium, the centriole at the base of the cilium is surrounded by the subdistal appendage/basal foot marker centriolin. Expansion microscopy - maximum zprojection of a confocal stack. Magenta: staining for acetylated tubulin. Green: staining for centriolin, marking subdistal appendages on the mother centriole at the base of the cilium.

791 Scale bar $=2 \mu \mathrm{m}$.

792 (E) In mature OSNs, a single cilium is surrounded by centriolin. Expansion microscopy - mature OSN imaged en face, maximum z-projection of a confocal stack. Magenta: staining for acetylated tubulin. Green: staining for centriolin. Arrow: centriolin surrounds the base of one cilium. Other cilia of the same dendrite are only associated with one centriolin punctum.

Figure 4-figure supplement 1. Centriole maturation in progenitors and migrating centriole Scale bar $=2 \mu \mathrm{m}$. groups.

(A) During centriole migration in immature OSNs, a single centriole bears distal and subdistal appendages. Expansion microscopy - single plane fluorescence image of 
centrioles below the apical surface in a side view of the expanded olfactory epithelium. Cyan: staining for ODF2, marking subdistal appendages. Yellow: staining for Cep164, marking distal appendages. Magenta: staining for acetylated tubulin. Within the group, a single centriole bears both ODF2 and Cep164. Scale bar $=2 \mu \mathrm{m}$.

(B) During centriole migration in immature OSNs, a single centriole bears centriolin.

808 Expansion microscopy - Single plane fluorescence image of centrioles below the apical 809 surface in expanded olfactory epithelium. Green: staining for centriolin. Magenta: staining for 810 acetylated tubulin. Scale bar $=2 \mu \mathrm{m}$.

811 (C) In progenitor cells, a single centriole bears distal and subdistal appendages. Expansion microscopy - Single plane fluorescence image of centriole rosettes in expanded olfactory epithelium. Cyan: staining for ODF2. Yellow: staining for CEP164. Magenta: staining for acetylated tubulin. Arrow: subdistal appendages and distal appendages are found on a single centriole. Scale bar $=2 \mu \mathrm{m}$. an MMCC imaged en face in expanded nasal epithelium. Green: staining for centriolin, marking a basal foot on each centriole. Magenta: staining for acetylated tubulin. Scale bars

\section{Figure 5. Centriole progression toward the apical surface can be altered by stabilizing}

\section{3 microtubules.}

824 (A) Schematic: workflow from sample collection through imaging of olfactory epithelium 825 explants. Septal olfactory epithelium was taken from adult mice expressing eGFP-centrin2 826 and Arl13b-mCherry and plated on transwell filters with an air-liquid interface. After 1 or 6 827 hours, samples were fixed and processed for sectioning. Stained sections were analyzed by 828 confocal microscopy. 
829 (B) Progenitor cells synthesize DNA by 1 hour ex vivo. Fluorescence image of an olfactory 830 epithelium explant grown in EdU for 1 hour, maximum intensity projection. Gray: DAPI, 831 marking all nuclei. Cyan: EdU, conjugated to dye after fixation, marks cells that synthesized 832 DNA ex vivo. Dashed line: the apical surface. Double solid line: basal lamina. Box: location 833 of the inset, B'. Scale bar $=10 \mu \mathrm{m}$. (B') Inset from panel B showing a progenitor cell positive 834 for EdU. Magenta: staining for $\beta$-tubulin III, showing that the cell is neuronally-fated. Yellow: 835 eGFP-centrin 2, showing signal consistent with centriole amplification. Cyan: EdU. Scale bar $836=2 \mu \mathrm{m}$.

837 (C) More cells synthesize DNA by 6 hours ex vivo. Fluorescence image of an olfactory 838 epithelium explant grown in EdU for 6 hours, maximum intensity projection. Tissue was taken from the same animal as that shown in panel B. Gray: DAPI, marking all nuclei. Cyan: EdU, conjugated to dye after fixation, shows an increased number of cells that have synthesized DNA ex vivo, compared to one hour treatment. Box: location of the inset, C'. Scale bar $=10 \mu \mathrm{m}$. (C') Inset from panel C showing a progenitor cell positive for EdU. Magenta: staining for $\beta$-tubulin III, showing that the cell is neuronally-fated. Yellow: eGFPcentrin 2 shows signal consistent with centriole amplification. Cyan: EdU. Scale bar $=2 \mu \mathrm{m}$.

(D) Control image of centriole group position in explants treated with DMSO for 6 hours. Single plane fluorescence image. Green: eGFP-centrin 2. Magenta: dye-conjugated phalloidin. Dashed line: apical surface. Arrows: migrating centriole groups. Scale bar $=10 \mu \mathrm{m}$.

(E) Centriole group position in explants treated with paclitaxel for 6 hours. Single plane fluorescence image. Tissue was taken from the same animal as that shown in Fig 5D.

(F) Bar plot summarizing centriole group position in explants grown for 1 or 6 hours in the 854 presence of DMSO or paclitaxel. Green bars: migrating centriole groups in the subapical 
compartment of the epithelium (defined in Fig 1A). Black bars: centriole groups in the middle and basal regions of the epithelium (defined in Fig 1A). Magenta circles: normalized number of groups for the female sample. Magenta squares: normalized number of groups for the male sample. Counts were normalized to the lateral length of the basal lamina. Length of epithelium scored for each timepoint: 1 hour DMSO $=988.17 \mu \mathrm{m}, 1$ hour paclitaxel $=905.37$ $\mu \mathrm{m}, 6$ hours DMSO $=1126.44 \mu \mathrm{m}, 6$ hours paclitaxel $=1019.76 \mu \mathrm{m} . \mathrm{N}=2$. See Figure $5-$ Source Data 1 for values. Figure 6. Summary of centriole migration and maturation during OSN differentiation.

864 Diagram summarizing the migration and maturation of centrioles over the course of OSN differentiation. A double solid line marks the basal lamina, and a dashed line is drawn above the apical surface. Newly-formed centrioles lacking appendages are represented as green dots, centrioles with appendages as magenta dots, and the parental centriole as a magenta dot with a yellow outline. After centriole amplification and mitosis in the progenitor, the immature OSN extends a dendrite toward the apical surface. Centrioles migrate primarily in groups during dendrite elongation. At the apical surface, a single cilium forms from the appendage-bearing,

871 parental centriole, before multiple cilia form in the mature OSN. 


\section{References}

876

877 Al Jord, A., Shihavuddin A., d’Aout R.S., Faucourt M., Genovesio A., Karaiskou A., SobczakThepot J., Spassky N., Meunier A. (2017). Calibrated mitotic oscillator drives motile ciliogenesis. Science 358(6364):803-806.

Anderson R.G.W., Brenner R.M. (1971). The formation of basal bodies (centrioles) in the rhesus monkey oviduct. J. Cell Biol. 50:10-34.

Arquint C., Nigg E.A. (2014). STIL microcephaly mutations interfere with APC/C-mediated degradation and cause centriole amplification. Curr Biol. 24(4):351-60.

Bangs, F.K., Schrode, N., Hadjantonakis, A.K., and Anderson, K. V. (2015). Lineage specificity of primary cilia in the mouse embryo. Nat. Cell Biol. 17, 113-122.

Baas, P.W., and Buster, D.W. (2004). Slow Axonal Transport and the Genesis of Neuronal Morphology. J. Neurobiol. 58, 3-17.

Baas, P.W., Rao, A.N., Matamoros, A.J., and Leo, L. (2016). Stability properties of neuronal microtubules. Cytoskeleton (Hoboken). 73, 442-460

Boixvieux-Ulrich E., Laine M-C, Sandoz D. (1989). In vitro effects of taxol on ciliogenesis in quail oviduct. J. Cell Sci. 92 ( Pt 1):9-20.

Bolivar J., Huynh J.R., Lopez-Schier H., Gonzalez C., St. Johnston D., Gonzalez-Reyes A. (2001). Centrosome migration into the Drosophila oocyte is independent of BicD and egl, and of the organisation of the microtubule cytoskeleton. Development 128(10):1889-97. Biogenesis. Annu Rev Biochem. 88:691-724.

897 Burakov, A. V., and Nadezhdina, E.S. (2020). Centering and Shifting of Centrosomes in Cells. Cells 9(6): 1351.

899 Burton, P.R. (1985). Ultrastructure of the olfactory neuron of the bullfrog: the dendrite and its 
microtubules. J. Comp. Neurol. 242, 147-160.

901 Cajanek L., and Nigg E.A. (2014). Cep164 triggers ciliogenesis by recruiting Tau tubulin kinase 2 to the mother centriole. Proc. Nat. Acad. Sci. 111 (28) E2841-E2850.

903 Challis R.C., Tian H., Wang J., He J., Jiang J., Chen X., Yin W., Connelly T., Ma L., Yu C.R.,

904 Pluznick J.L., Storm D.R., Huang L., Zhao K., Ma M. (2015). An Olfactory Cilia Pattern in 905 the Mammalian Nose Ensures High Sensitivity to Odors. Curr Biol. 25(19):2503-12.

906 Ching, K., and Stearns, T. (2020). Centrioles are amplified in cycling progenitors of olfactory sensory neurons. PLoS Biol. 18, 1-17.

Choi Y-W., Liu P., Sze S.K., Dai C., Qi R.Z. (2010). CDK5RAP2 stimulates microtubule nucleation by the y-tubulin ring complex. J. Cell Biol. 191 (6): 1089-1095.

Cuschieri A., Bannister L.H. (1975). The development of the olfactory mucosa in the mouse: Electron microscopy. J. Anat. 119:471-498.

Dawe, H. R., Farr, H., \& Gull, K. (2007). Centriole/basal body morphogenesis and migration during ciliogenesis in animal cells. Journal of Cell Science, 120(1), 7-15.

Delaval B., Doxsey S.J. (2010). Pericentrin in cellular function and disease. J. Cell Biol. 188(2): 181-190.

Farbman, A. I. (1977). Differentiation of olfactory receptor cells in organ culture. The Anatomical Record, 189(2), 187-199.

Fong K-W., Choi Y-W., Rattner J.B., Qi R.Z. (2008). CDK5RAP2 is a pericentriolar protein that functions in centrosomal attachment of the gamma-tubulin ring complex. Mol. Biol. Cell. 19(1):115-25.

Gambarotto, D., Zwettler, F.U., Le Guennec, M., Schmidt-Cernohorska, M., Fortun, D., Borgers,

924 Hatch, E.M., Kulukian, A., Holland, A.J., Cleveland, D.W., and Stearns, T. (2010). Cep152 interacts with PIk4 and is required for centriole duplication. J. Cell Biol. 191, 721-729. 
926 Heist, H.E., and Mulvaney, B.D. (1968). Centriole migration. J Ultrastruct Res 24, 86-101.

927 Izquierdo D., Wang W-J, Uryu K., Tsou M.F.B. (2014). Stabilization of cartwheel-less centrioles for duplication requires CEP295-mediated centriole-to-centrosome conversion. Cell Rep.

Jain R., Pan J., Driscoll J.A., Wisner J.W., Huang T., Gunsten S.P., You Y., Brody S.L. (2010). Temporal Relationship between Primary and Motile Ciliogenesis in Airway Epithelial

Jenkins P.M., McEwen, D.P., Martens, J.R. (2009). Olfactory Cilia: Linking Sensory Cilia Function and Human Disease. Chem Senses, 34(5):451-464.

Jurczyk A., Gromley A., Redick S., San Agustin J., Witman G., Pazour G.J., Peters D.J.M., Doxsey S. (2004). Pericentrin forms a complex with intraflagellar transport proteins and polycystin-2 and is required for primary cilia assembly. J. Cell Biol. 166(5): 637-643.

Klimenkov, I. V., Sudakov, N.P., Pastukhov, M. V., Svinov, M.M., and Kositsyn, N.S. (2018). Rearrangement of Actin Microfilaments in the Development of Olfactory Receptor Cells

King S.J., Schroer T.A. (1999). Dynactin increases the processivity of the cytoplasmic dynein

Kong D., Farmer V., Shukla A., James J., Gruskin R., Kiriyama S., Loncarek J. (2014). Centriole maturation requires regulated Plk1 activity during two consecutive cell cycles. J. Cell Biol. 206(7):855-65.

Kulaga, H.M., Leitch, C.C., Eichers, E.R., Badano, J.L., Lesemann, A., Hoskins, B.E., Lupski, neurons lack centrosomal $ү$ - tubulin. Neurosci. Lett. 229, 17-20. 
Lemullois M., Boisvieux-Ulrich E., Laine M-C, Chailley B., Sandoz D. (1988). Development and functions of the cytoskeleton during ciliogenesis in metazoa. Biology of the Cell. 63,195-

Lee Y.L., Santé J., Comerci C.J., Cyge B., Menezes L.F., Li F.Q., Germino G.G., Moerner W.E., Takemaru K., Stearns T. (2014) Cby1 promotes Ahi1 recruitment to a ring-shaped domain at the centriole-cilium interface and facilitates proper cilium formation and

Li, W., Yi, P., Zhu, Z., Zhang, X., Li, W., and Ou, G. (2017). Centriole translocation and degeneration during ciliogenesis in Caenorhabditis elegans neurons. EMBO J.

Liang, F. (2018). Olfactory receptor neuronal dendrites become mostly intra-sustentacularly enwrapped upon maturity. J. Anat. 232, 674-685.

Liang, X., Kokes, M., Fetter, R.D., Sallee, M.D., Moore, A.W., Feldman, J.L., and Shen, K. (2020). Growth cone-localized microtubule organizing center establishes microtubule orientation in dendrites. Elife 9,1-28.

Liu, Z., Nguyen, Q. P. H., Nanjundappa, R., Delgehyr, N., Megherbi, A., Doherty, R., Thompson, J., Jackson, C., Albulescu, A., Heng, Y. M., Lucas, J. S., Dell, S. D., Meunier, A., Czymmek, K., Mahjoub, M. R., \& Mennella, V. (2020). Super-Resolution Microscopy and patterns identify mouse cilia genes. Physiological Genomics, 32(2), 198-206.

977 Menco, B. P. M., Leunissen, J. L. M., Bannister, L. H., \& Dodd, G. H. (1978). Bovine olfactory 
and nasal respiratory epithelium surfaces - High-voltage and scanning electron microscopy, and cryo-ultramicrotomy. Cell and Tissue Research, 193(3), 503-524.

Menco BP (1997) Ultrastructural aspects of olfactory signaling. Chem Senses 22: 295-311

McEwen DP, Koenekoop RK, Khanna H, Jenkins PM, Lopez I, Swaroop A, Martens JR. (2007). of G proteins in cilia of olfactory sensory neurons. Proc Natl Acad Sci USA.104:1591715922.

McEwen D.P., Jenkins P.M., Martens J.R. (2008). Olfactory Cilia: Our Direct Neuronal Connection to the External World. Curr. Top. Dev. Biol. 85:333-370.

McIntyre, J.C., Titlow, W.B., and McClintock, T.S. (2010). Axon Growth and Guidance Genes Identify Nascent, Immature, and Mature Olfactory Sensory Neurons. J Neurosci Res 88, 3243-3256.

Moulton, D. G., \& Beidler, L. M. (1967). "Physiological Reviews.” The American Physiological Society, 47(4), 1-52.

Mulvaney, B.D., and Heist, H.E. (1971). Centriole migration during regeneration and normal development of olfactory epithelium. J. Ultrasructure Res. 35, 274-281.

994 Nigg E.A., Stearns T. (2011). The centrosome cycle: Centriole biogenesis, duplication and inherent asymmetries. Nat. Cell Biol. 13(10):1154-60.

Oberland, S., and Neuhaus, E.M. (2014). Whole Mount Labeling of Cilia in the Main Olfactory System of Mice. J. Vis. Exp. 6-11.

Presley J.F., Cole N.B., Schroer T.A., Hirschberg K., Zall K.J.M., Lippincott-Schwartz J. (1997). ER-to-Golgi transport visualized in living cells. Nature 389, 81-85.

Rodriguez-Gil, D.J., Bartel, D.L., Jaspers, A.W., Mobley, A.S., Imamura, F., and Greer, C.A. (2015). Odorant receptors regulate the final glomerular coalescence of olfactory sensory neuron axons. Proc. Natl. Acad. Sci. 112, 5821-5826.

Roy S. (2020). Finding order in slow axonal transport. Current Opinion in Neurobiology 63, 87- 
94.

Sahabandu N., Kong D., Magidson V., Nanjundappa R., Sullenberger C., Mahjoub M.R., Loncarek J. (2019). Expansion microscopy for the analysis of centrioles and cilia. J Microsc. 276(3):145-149.

Schindelin, J., Arganda-Carreras, I., Frise, E., Kaynig, V., Longair, M., Pietzsch, T., Preibisch, S., Rueden, C., Saalfeld, S., Schmid, B., et al. (2012). Fiji: An open-source platform for biological-image analysis. Nat. Methods 9, 676-682.

Shukla A., Kong D., Sharma M., Magidson V., Loncarek J. (2015). Plk1 relieves centriole block to reduplication by promoting daughter centriole maturation. Nat. Comm. 6:8077.

Sorokin S.P. (1968). Reconstructions of centriole formation and ciliogenesis in mammalian lungs. J. Cell Sci. 3,207-230.

Stiess, M., Maghelli, N., Kapitein, L.C., Gomis-Ruth, S., Wilsch-Bräuninger, M., Hoogenraad, C.C., Tolic-Norrelykke, I.M., and Bradke, F. (2010). Axon Extension Occurs Independently of Centrosomal Microtubule Nucleation. Science. 327, 704-707.

Strnad P., Leidel S., Vinogradova T., Euteneuer U., Khodjakov A., Gonczy P. (2007). Regulated HsSAS-6 Levels Ensure Formation of a Single Procentriole per Centriole during the Centrosome Duplication Cycle. Dev. Cell 13(2)203-213.

Schwarzenbacher, K., Fleischer, J., \& Breer, H. (2005). Formation and maturation of olfactory cilia monitored by odorant receptor-specific antibodies. Histochemistry and Cell Biology, 123(4-5), 419-428. centriole migration. J Cell Sci 89 (1): 67-80.

Tanos B.E., Yang H-J., Soni R., Wang W-J., Macaluso F.P., Asara J.M., Tsou M-F B. (2013). 
Centriole distal appendages promote membrane docking, leading to cilia initiation. Genes and Dev. 2013. 27: 163-168.

Uytingco C.R., Williams C.L., Xie C., Shively D.T., Green W.W., Ukhanov K., Zhang L., Nishimura D.Y., Sheffield V.C., Martens J.R. (2019). BBS4 is required for intraflagellar transport coordination and basal body number in mammalian olfactory cilia. J. Cell Sci. 132(5):jcs222331.

Ukhanov K., Uytingco C., Green W., Zhang L., Schurmans S., Martens J. (2022). INPP5E controls ciliary localization of phospholipids and the odor response in olfactory sensory neurons. J. Cell Sci. 135(5):jcs258364.

Vladar E.K., Bayly R.D., Sangoram A., Scott M.P., Axelrod J.D. (2012). Microtubules Enable the Planar Cell Polarity of Airway Cilia. Curr Biol. 22(23):2203-12.

Vlijm, R., Li, X., Panic, M., Rüthnick, D., Hata, S., Herrmannsdörfer, F., Kuner, T., Heilemann, M., Engelhardt, J., Hell, S.W., et al. (2018). STED nanoscopy of the centrosome linker reveals a CEP68-organized, periodic rootletin network anchored to a C-Nap1 ring at centrioles. Proc. Natl. Acad. Sci. U. S. A. 201716840.

Williams, C. L., Mclntyre, J. C., Norris, S. R., Jenkins, P. M., Zhang, L., Pei, Q., .. Martens, J. R. (2014). Direct evidence for BBSome-associated intraflagellar transport reveals distinct properties of native mammalian cilia. Nature Communications, 5, 5813.

Wilkes, O.R., and Moore, A.W. (2020). Distinct microtubule organizing center mechanisms combine to generate neuron polarity and arbor complexity. Front. Cell. Neurosci. $19 ; 14: 594199$.

Wang W-J, Soni R.K., Uryu K., Tsou M.F.B. (2011). The conversion of centrioles to centrosomes: essential coupling of duplication with segregation. J. Cell Biol. 193(4): 727-739.

Yang, J., Liu, X., Yue, G., Adamian, M., Bulgakov, O., and Li, T. (2002). Rootletin, a novel coiled-coil protein, is a structural component of the ciliary rootlet. J. Cell Biol. 159, 431- 
440.

1057 Ying G., Avasthi P., Irwin M., Gerstner C.D., Frederick J.M., Lucero M.T., Baehr W. (2014).

1058 Centrin 2 Is Required for Mouse Olfactory Ciliary Trafficking and Development of

1059 Ependymal Cilia Planar Polarity. J. Neurosci. 34(18): 6377-6388.

1060 Yonezawa S., Shigematsu M., Hirata K., Hayashi K. (2015). Loss of y-tubulin, GCP-WD/NEDD1

1061 and CDK5RAP2 from the Centrosome of Neurons in Developing Mouse Cerebral and

1062 Cerebellar Cortex. Acta Histochem Cytochem.48(5): 145-152.

1063 Zhao H., Chen Q., Li F., Cui L., Xie L., Huang Q., Liang X., Zhou J., Yan X., Zhu X. (2021).

1064 Fibrogranular materials function as organizers to ensure the fidelity of multiciliary

1065 assembly. Nat Comm. 12(1):1273.

1066 Zhou R., Liu H., Ju T., Dixit R. (2020). Quantifying the polymerization dynamics of plant cortical

1067 microtubules using kymograph analysis. Methods Cell Biol.160:281-293. 


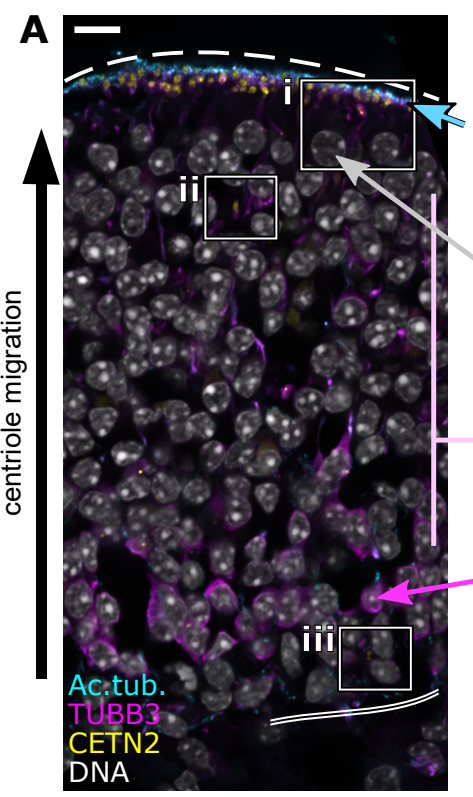

B

\section{CETN2}

\section{actin}

C

sustentacular cell

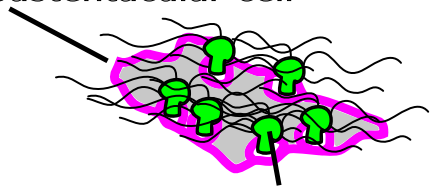

OSN dendrite

E

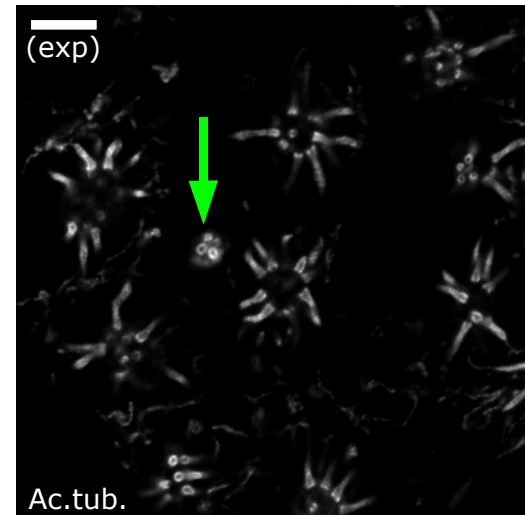

mature OSN cilia
at apical surface

sustentacular cell nucleus
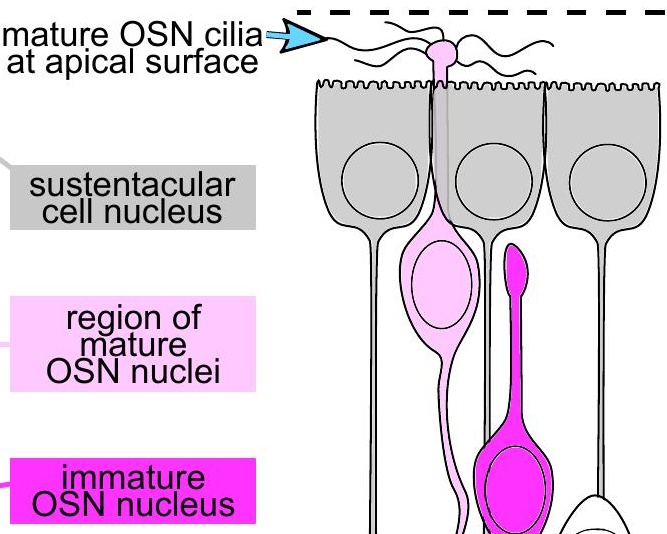

basal lamina

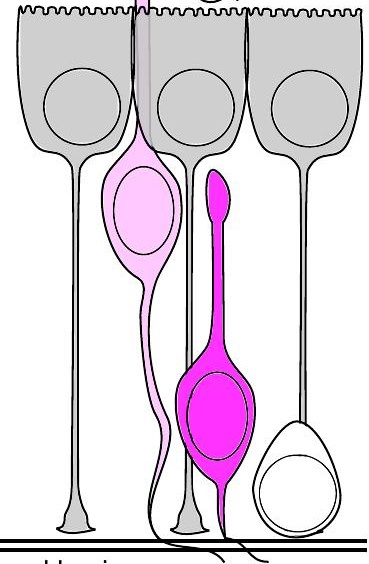

D

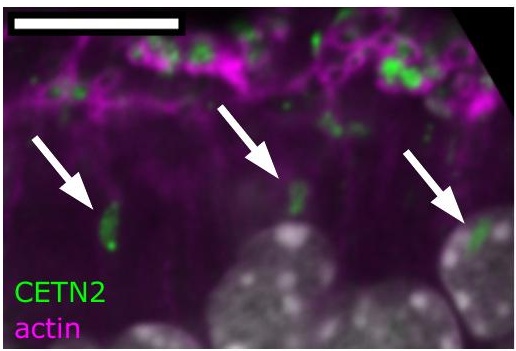

F

(exp)

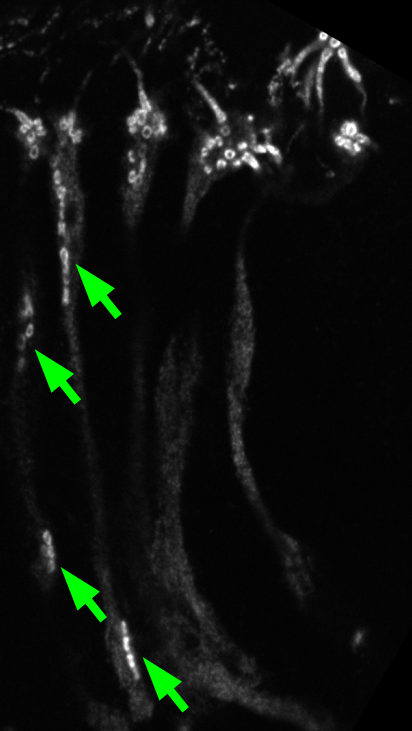

Ac.tub. 
Figure 1 - Supplement 1

A $i$

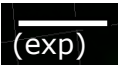

$i \overline{(\exp )}$

\begin{tabular}{l|l|l|}
\hline 0 & Position Z & 6.13
\end{tabular}

Ac.tub.

B i
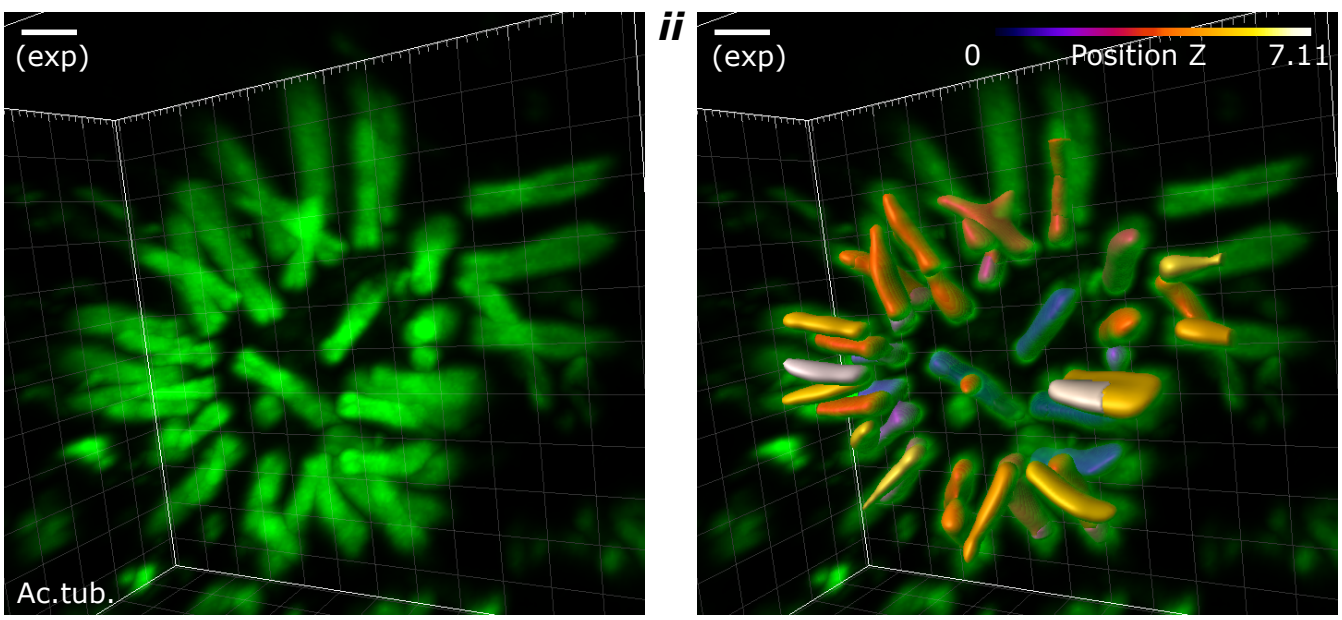

C

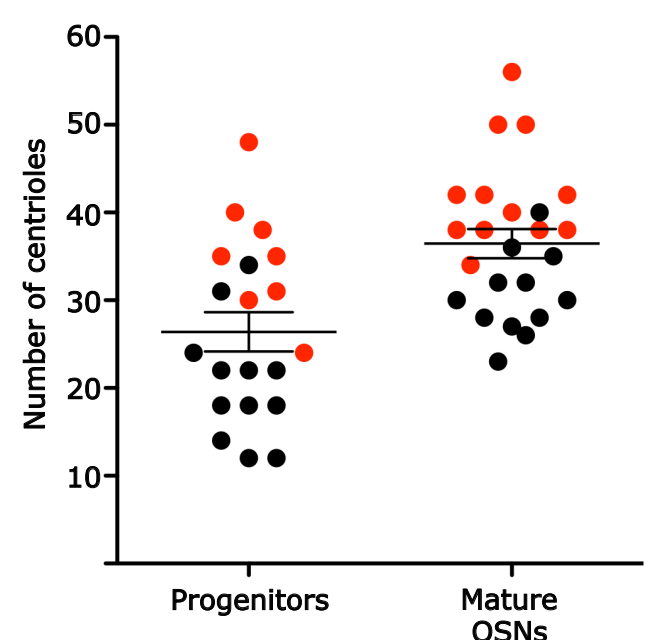

D

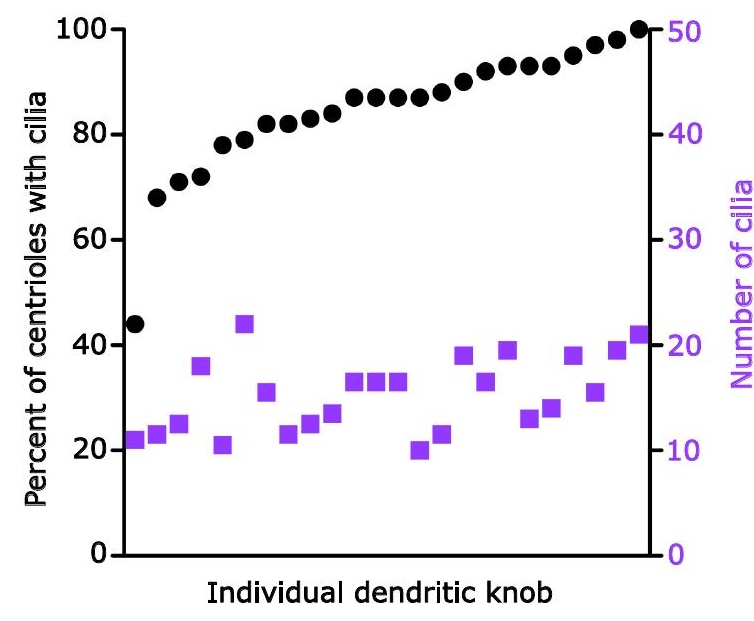


A

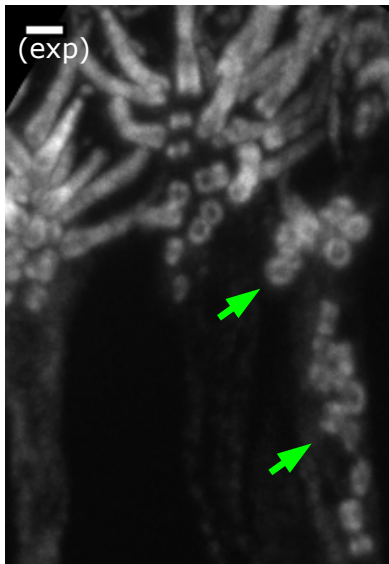

Ac.tub.
B

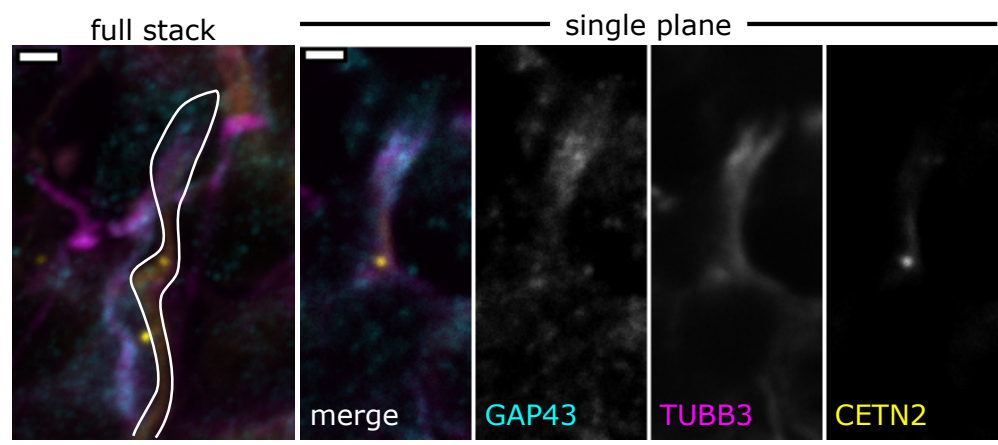

C i ${ }_{\text {CETN2, DNA }}$

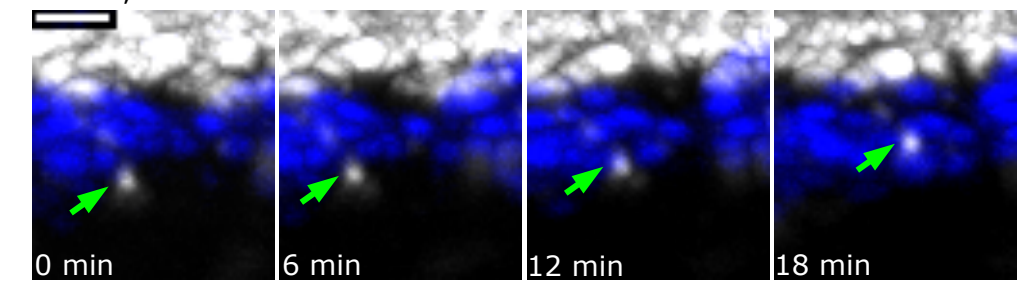

ij Time

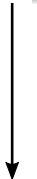

Apical

Basal

Di CETn2, DNA
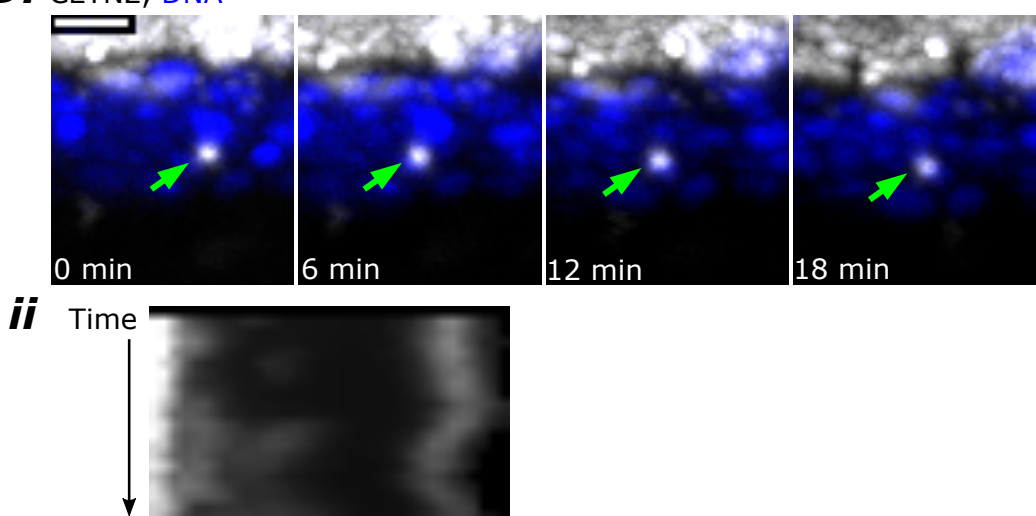

Apical 
A $\overline{(\exp )}$

CEP164 Ac.tub. centrin DNA

:5

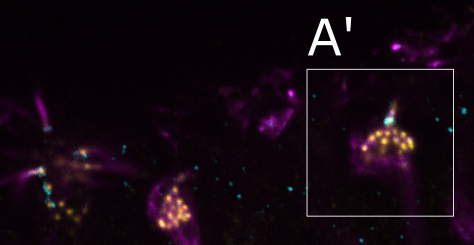

$A^{\prime}$ immature

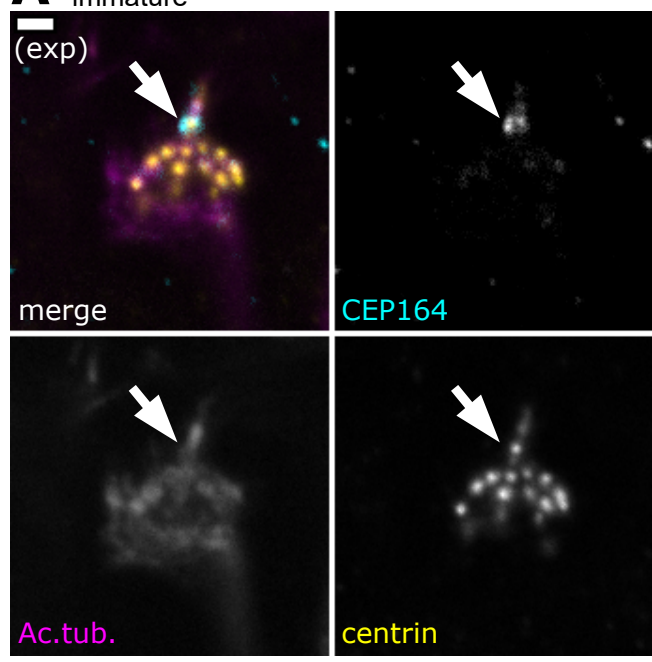

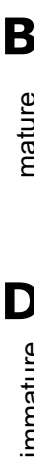
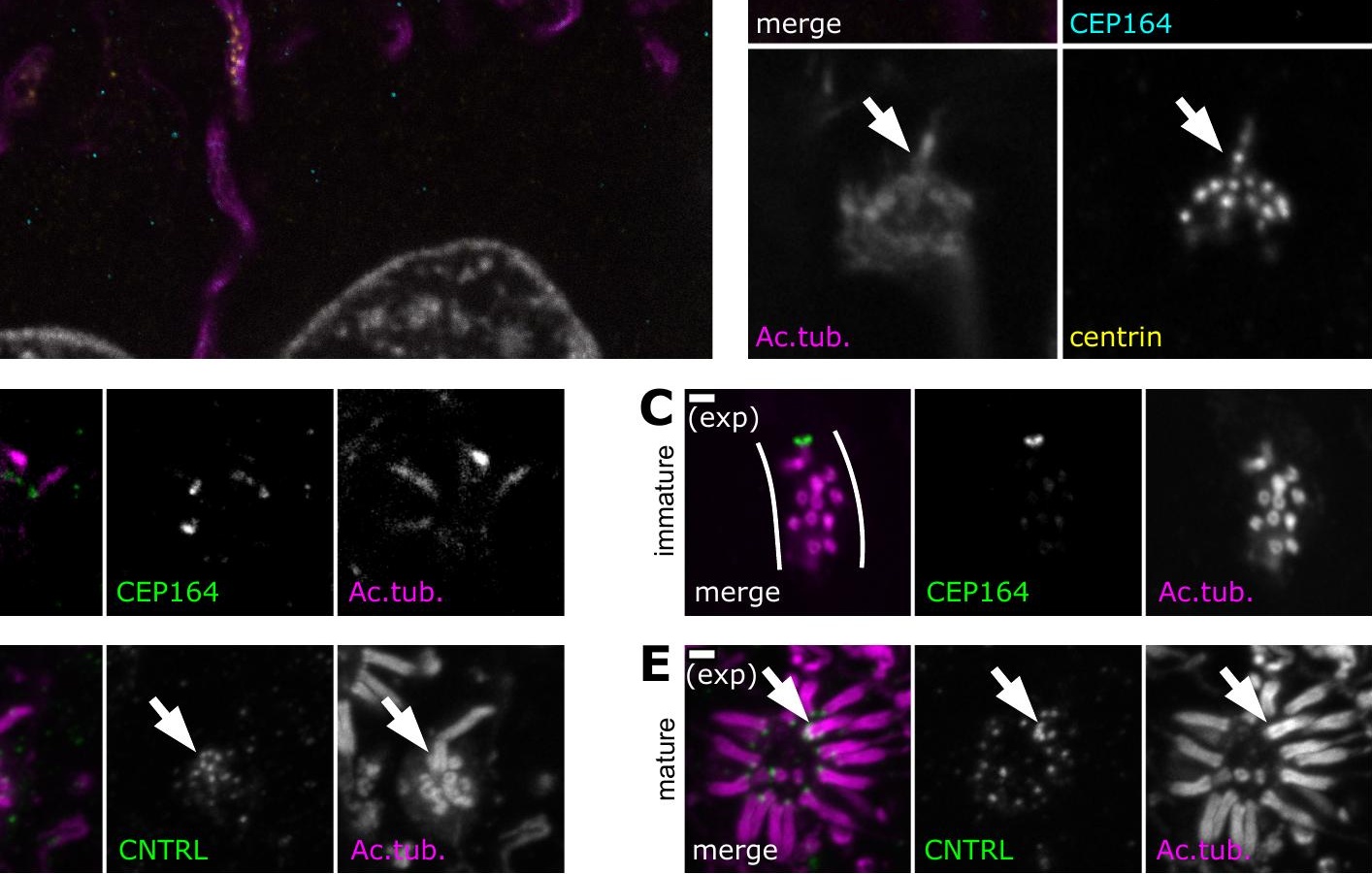
Figure 4 - Supplement 1
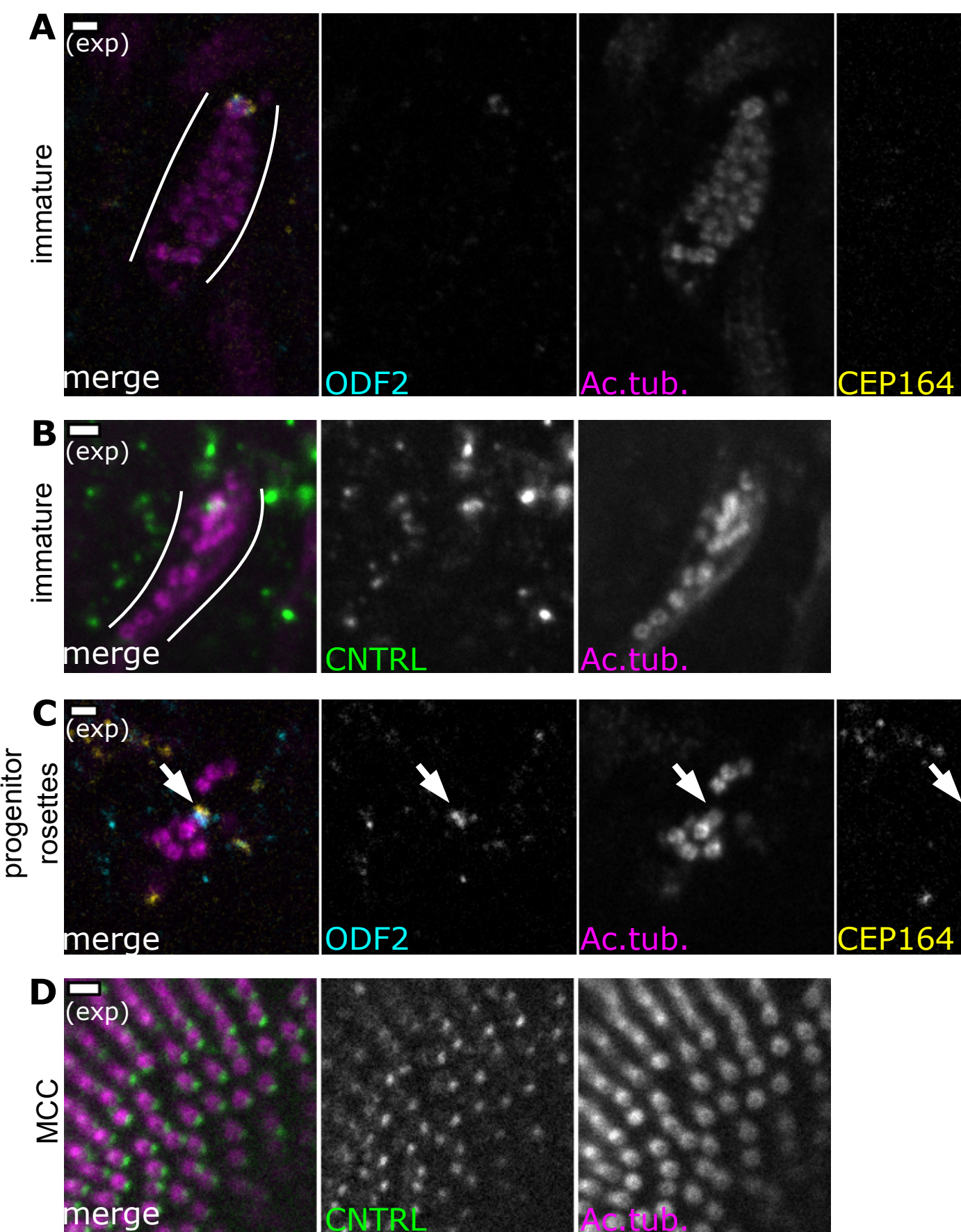
$\mathbf{A}$

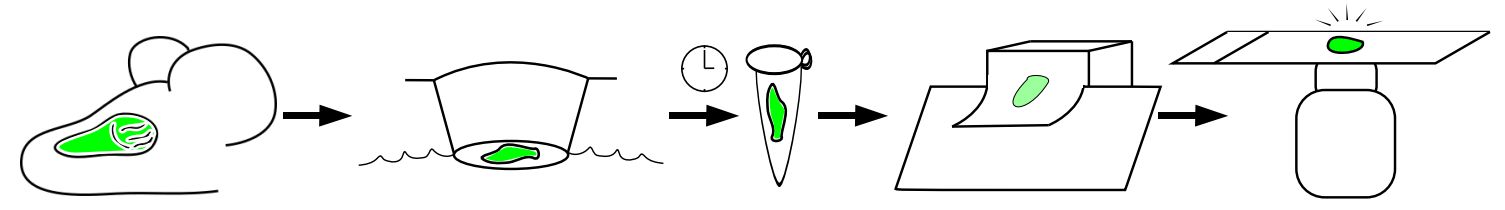

B

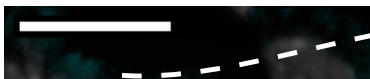

- DNA
EdU

ARL13B

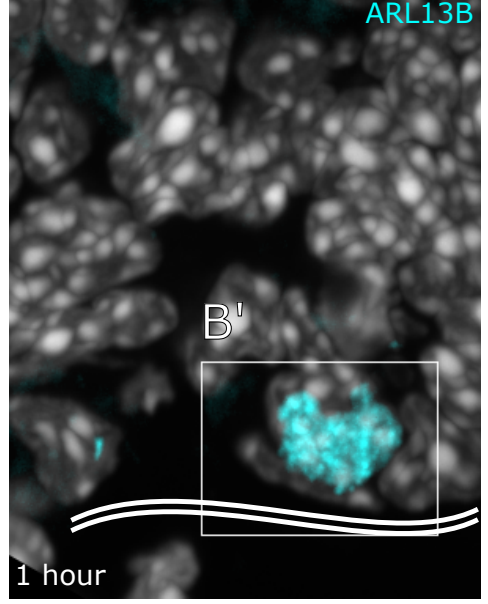

D

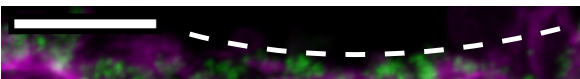

CETN2

actin

6 hours DMSO

$\mathbf{E}$

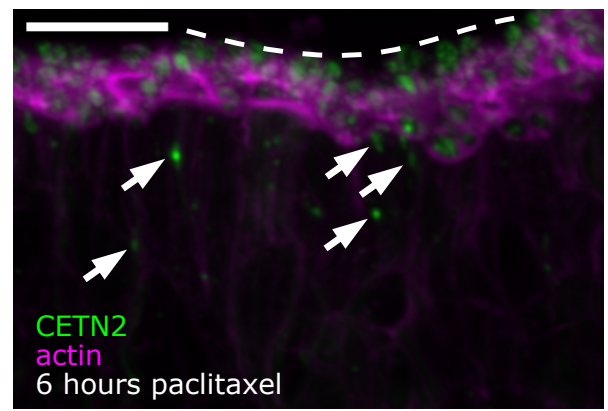

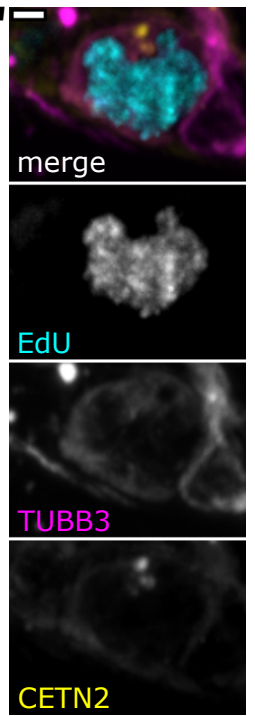

F
C

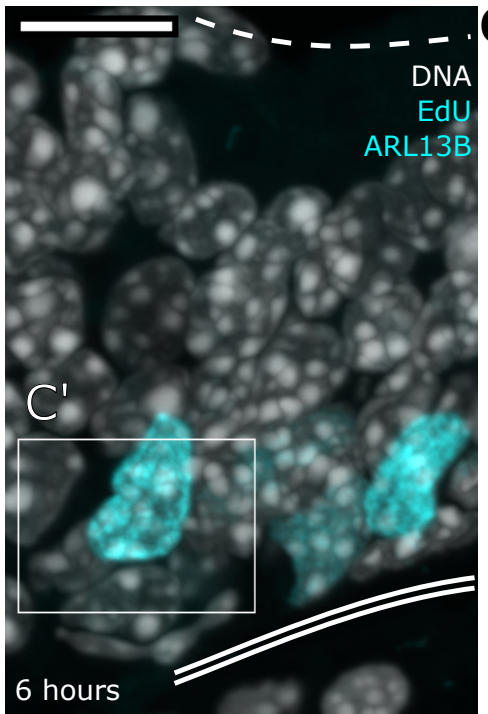

6

- number of middle and basal centriole groups

$\square$ number of subapical

centriole groups

응 4

ฮั

응

윽 3

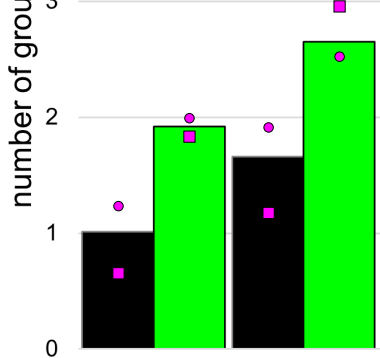

1 hour 1 hour

DMSO paclitaxel

DI

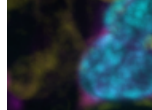

merge

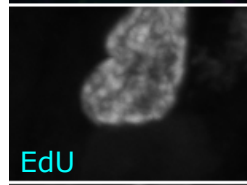

TUBB3

CETN2

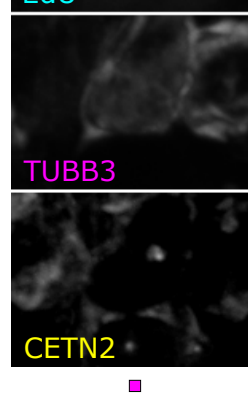

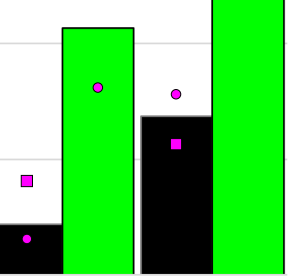

6 hours 6 hours

DMSO paclitaxel 


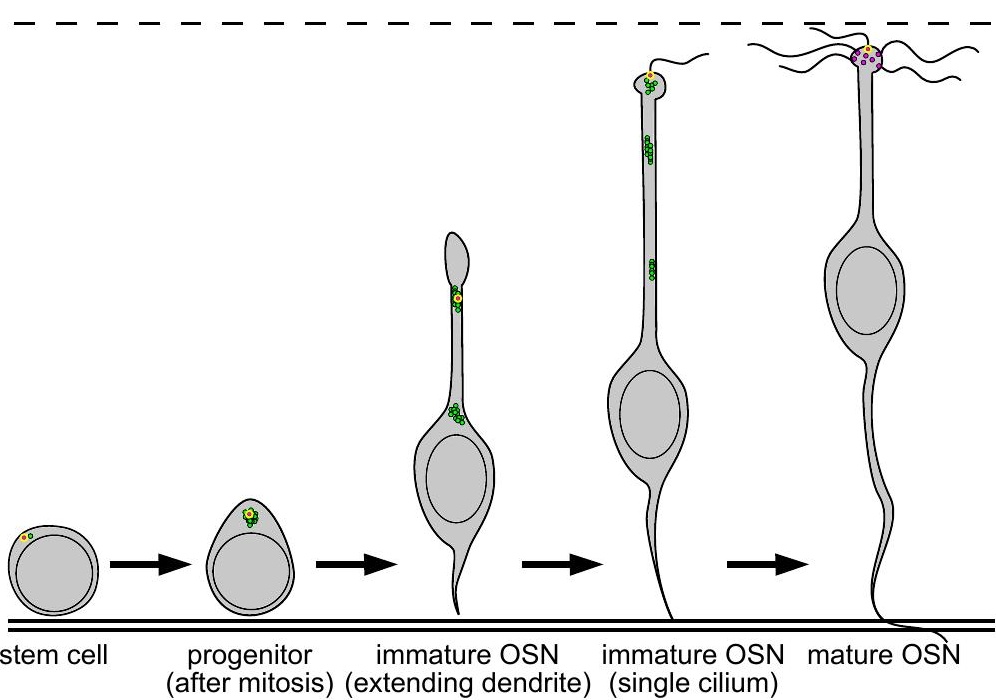

